\title{
Vertex algebras and vertex Poisson algebras
}

\author{
Haisheng $\operatorname{Li}^{1}$ \\ Department of Mathematical Sciences, Rutgers University, Camden, NJ 08102 \\ and \\ Department of Mathematics, Harbin Normal University, Harbin, China
}

\begin{abstract}
This paper studies certain relations among vertex algebras, vertex Lie algebras and vertex Poisson algebras. In this paper, the notions of vertex Lie algebra (conformal algebra) and vertex Poisson algebra are revisited and certain general construction theorems of vertex Poisson algebras are given. A notion of filtered vertex algebra is formulated in terms of a notion of good filtration and it is proved that the associated graded vector space of a filtered vertex algebra is naturally a vertex Poisson algebra. For any vertex algebra $V$, a general construction and a classification of good filtrations are given. To each $\mathbb{N}$-graded vertex algebra $V=\coprod_{n \in \mathbb{N}} V_{(n)}$ with $V_{(0)}=\mathbb{C} 1$, a canonical (good) filtration is associated and certain results about generating subspaces of certain types of $V$ are also obtained. Furthermore, a notion of formal deformation of a vertex (Poisson) algebra is formulated and a formal deformation of vertex Poisson algebras associated with vertex Lie algebras is constructed.
\end{abstract}

\section{Introduction}

In quantum physics, a very important role is played by Poisson algebras. A Poisson algebra structure on a vector space $A$ is a combination of a commutative associative algebra structure and a Lie algebra structure with a certain compatibility condition (the Leibniz rule). Important examples from quantum mechanics are those with $A=C^{\infty}(M)$, where $M$ are symplectic manifolds (the phase spaces of dynamical systems). Another important family of Poisson algebras are the Poisson algebras $S(\mathfrak{g})\left(=\mathbb{C}\left[\mathfrak{g}^{*}\right]\right)$ associated with (finite-dimensional) Lie algebras $\mathfrak{g}$. In the Poisson world, among the most important issues is the algebraic deformation quantization of Poisson algebras (see [BFFLS], [Ko]).

Vertex (operator) algebras are known as the mathematical counterparts of chiral algebras in two-dimensional quantum conformal field theory and are analogous to associative algebras in certain aspects. (At the same time, in many aspects vertex (operator) algebras are also analogous to both Lie algebras and commutative associative algebras.) One example of such analogy is that Borcherds' commutator formula, which is just part of the vertex algebra structure, gives rise to a certain Lie algebra (cf. [B1], [FFR], [Li1], [MP]), just as the commutator of an associative algebra gives rise to a canonical Lie algebra. This essentially motivated the introduction of Lie algebra analogues of vertex algebras. In $[\mathrm{K}]$, among other things Kac introduced a notion of conformal algebra and independently Primc introduced and studied a notion of vertex Lie algebra in $[\mathrm{P}]$. (A different

\footnotetext{
${ }^{1}$ Partially supported by NSF grant DMS-9970496 and a grant from Rutgers Research Council
} 
notion of vertex Lie algebra was introduced in [DLM2].) As it was explained in Remark 2.6, the notion of conformal algebra and the notion of vertex Lie algebra are equivalent. Following $[\mathrm{FB}]$ we use conformal algebra and vertex Lie algebra synonymously in this paper. The main axiom defining the notion of vertex Lie algebra is what Primc called "half commutator formula." Associated to each vertex Lie algebra $R$, there is an honest Lie algebra $\mathcal{L}(R)$, the underlying vector space of which is a certain quotient space of the loop space $L(R)\left(=R \otimes \mathbb{C}\left[t, t^{-1}\right]\right)$ of $R$. Just as one gets the universal enveloping algebra $U(\mathfrak{g})$ (an associative algebra) from any Lie algebra $\mathfrak{g}$, one gets a canonical vertex algebra $\mathcal{V}(R)$ (see [DLM2], [FB], [K], [P]) from any vertex Lie algebra $R$, where the vertex algebra $\mathcal{V}(R)$ is a suitably defined Verma $\mathcal{L}(R)$-module.

With the notion of vertex Lie algebra, one naturally arrives at the notion of vertex Poisson algebra. A vertex Poisson algebra structure (see [FB]; cf. [BD], [EF], [DLM2]) is a combination of a commutative vertex algebra structure (or equivalently a differential algebra structure) and a vertex Lie algebra structure with a natural compatibility condition. Vertex Poisson algebras were studied in [FB] (Chapter 15) and many important results were obtained. Among those results, it was noticed that the symmetric algebra of any vertex Lie algebra is naturally a vertex Poisson algebra and certain vertex Poisson algebras associated with affine and the Virasoro Lie algebras were realized as classical limits of vertex algebras. Certain connections between the classical and the quantum Drinfeld-Sokolov reductions were also exhibited.

In this paper, we shall study the connection of vertex algebras, vertex Lie algebras and vertex Poisson algebras in a more systematic way and the main purpose of this paper is to lay the foundation for future studies on vertex algebras and vertex Poisson algebras. As one of our main results, we introduce and study a notion of filtered vertex algebra and we show that the graded vector space of a filtered vertex algebra is naturally a vertex Poisson algebra. For any vertex algebra $V$, we give a general construction and a classification of filtered vertex algebras $(V, E)$ and we associate a canonical filtered vertex algebra to every $\mathbb{N}$-graded vertex algebra $V$ with $V_{(0)}=\mathbb{C} \mathbf{1}$. The introduction of the notion of filtered vertex algebra was motivated by certain classical results and certain results in [KL] on generating subspaces of a certain type of vertex operator algebras. In the classical case, the standard filtration on the universal enveloping algebra of a Lie algebra and the fundamental Poincare-Birkhoff-Witt theorem are closely related. Similarly, the canonical filtration we constructed is closely related to what we call generating subspaces with PBW spanning property and certain basic results are obtained.

For determining vertex Poisson algebra structures on a given differential algebra we give two general construction theorems of vertex Poisson algebras. Such construction theorems are very effective to determine whether a certain vertex Poisson algebra structure exists on a differential algebra. In particular, our construction theorems can be applied to show that there exists a natural vertex Poisson algebra structure on the symmetric algebra $S(R)$ of a vertex Lie algebra $R$ (Proposition 3.7; see also [FB], Example 15.2.2). As it is needed in the proof of the first construction theorem, we prove that in a suitable setting, the half commutator formula has $S_{3}$-symmetry. This makes vertex Lie algebras virtually look like Lie algebras. The proof of this particular result is based on certain 
results of $[\mathrm{P}]$ and $[\mathrm{FHL}]$.

For any vertex Lie algebra $R$, we construct a canonical map from the symmetric algebra $S(R)$ onto $\mathcal{V}(R)$, which is analogous to the canonical symmetrization map from $S(\mathfrak{g})$ to $U(\mathfrak{g})$ for a Lie algebra $\mathfrak{g}$, and we show that the vertex Poisson algebra $S(R)$ is isomorphic to $\operatorname{gr}_{E} \mathcal{V}(R)$, where $E$ is the associated canonical filtration. Motivated by a result of Frenkel and Ben-Zvi [FB], we formulate a notion of $*$-deformation of a vertex Poisson algebra and we construct a $*$-deformation of the vertex Poisson algebras associated with filtered vertex algebras and with vertex Lie algebras.

As with Poisson algebras, a fundamental problem is about the existence, uniqueness and construction of each vertex Poisson algebra. In a sequel we shall study the existence of $*$-deformation of a vertex Poisson structure on a free differential algebra (a polynomial algebra) and we shall use the vertex Poisson algebra $\operatorname{gr}_{E} V$ to study the algebraic structures of a vertex algebra $V$.

In this work we use many of Primc's results and ideas from $[\mathrm{P}]$ and we certainly use many ideas from [DLM2]. We benefit very much from reading Chapter 15 of [FB] and from talking with Ping Xu.

This paper is organized as follows: In Section 2, we review and discuss the notion of vertex Lie algebra (conformal algebra) and we present some new results. In Section 3, we recall the notion of vertex Poisson algebra and give two general construction theorems of vertex Poisson algebras. In Section 4, we study filtered vertex algebras. In Section 5, we study $*$-deformations of vertex (Poisson) algebras.

\section{Vertex algebras and vertex Lie algebras}

In this section we review and discuss the notions of vertex algebra and vertex Lie algebra (conformal algebra), and their relations. We recall certain results of Primc about the half commutator formula and the half Jacobi identity and we prove that the half Jacobi identity and the half commutator formula have $S_{3}$-symmetry. We also prove a result analogous to the classical result about the canonical (symmetrization) map from $S(\mathfrak{g})$ to $U(\mathfrak{g})$ for a Lie algebra $\mathfrak{g}$.

In this paper we use the standard formal variable notations and conventions as defined in $[\mathrm{FLM}]$ and [FHL] (cf. [LL]). In addition to the standard notations $\mathbb{C}$ and $\mathbb{Z}$, we use notations $\mathbb{N}$ for the set of nonnegative integers and $\mathbb{Z}_{+}$for the set of positive integers.

Let $V$ be any vector space. Following [P], for a formal series

$$
f\left(x_{1}, \ldots, x_{n}\right)=\sum_{m_{1}, \ldots, m_{n} \in \mathbb{Z}} u\left(m_{1}, \ldots, m_{n}\right) x_{1}^{-m_{1}-1} \cdots x_{n}^{-m_{n}-1} \in V\left[\left[x_{1}^{ \pm 1}, \ldots, x_{n}^{ \pm 1}\right]\right],
$$

we set

$$
\operatorname{Sing} f\left(x_{1}, \ldots, x_{n}\right)=\sum_{m_{1}, \ldots, m_{n} \geq 0} u\left(m_{1}, \ldots, m_{n}\right) x_{1}^{-m_{1}-1} \cdots x_{n}^{-m_{n}-1} .
$$

Clearly, for $1 \leq i \leq n$,

$$
\frac{\partial}{\partial x_{i}} \operatorname{Sing} f\left(x_{1}, \ldots, x_{n}\right)=\operatorname{Sing} \frac{\partial}{\partial x_{i}} f\left(x_{1}, \ldots, x_{n}\right) .
$$


For any nonempty subset $S=\left\{i_{1}, \ldots, i_{k}\right\}$ of $\{1, \ldots, n\}$, a formal series $f\left(x_{1}, \ldots, x_{n}\right)$ can be naturally viewed as a formal series $\tilde{f}\left(x_{i_{1}}, \ldots, x_{i_{k}}\right)$ in variables $x_{i_{1}}, \ldots, x_{i_{k}}$ with coefficients in the vector space $V\left[\left[x_{j}^{ \pm 1} \mid j \notin S\right]\right]$. Then we define

$$
\operatorname{Sing}_{x_{i_{1}}, \ldots, x_{i_{k}}} f\left(x_{1}, \ldots, x_{n}\right)=\operatorname{Sing} \tilde{f}\left(x_{i_{1}}, \ldots, x_{i_{k}}\right) .
$$

In particular, we have

$$
\operatorname{Sing}_{x_{1}} f\left(x_{1}, \ldots, x_{n}\right)=\sum_{m \in \mathbb{N}, m_{2}, \ldots, m_{n} \in \mathbb{Z}} u\left(m, m_{2}, \ldots, m_{n}\right) x_{1}^{-m-1} x_{2}^{-m_{2}-1} \cdots x_{n}^{-m_{n}-1} .
$$

Then

$$
\operatorname{Sing} f\left(x_{1}, \ldots, x_{n}\right)=\operatorname{Sing}_{x_{1}} \cdots \operatorname{Sing}_{x_{n}} f\left(x_{1}, \ldots, x_{n}\right) .
$$

The following result is due to Primc in $[\mathrm{P}]$ :

Lemma 2.1 Let $V$ be a vector space and let

$$
B \in V\left(\left(x_{1}, \ldots, x_{n}\right)\right), P \in(\text { End } V)\left[\left[x_{1}, \ldots, x_{n}\right]\right] .
$$

Then

$$
\operatorname{Sing}(P \cdot \operatorname{Sing}(B))=\operatorname{Sing}(P B) .
$$

In this paper we use the following definition of the notion of vertex algebra as in [LL]:

Definition 2.2 A vertex algebra is a vector space $V$ equipped with a linear map

$$
\begin{aligned}
Y: V & \rightarrow \operatorname{Hom}(V, V((x))) \\
v & \mapsto Y(v, x)=\sum_{n \in \mathbb{Z}} v_{n} x^{-n-1} \quad\left(\text { where } v_{n} \in \operatorname{End} V\right)
\end{aligned}
$$

and equipped with a distinguished vector $\mathbf{1} \in V$, called the vacuum vector, such that the following axioms hold:

$$
Y(\mathbf{1}, x)=1
$$

for $v \in V$,

$$
Y(v, x) \mathbf{1} \in V[[x]] \quad \text { and }\left.\quad Y(v, x) \mathbf{1}\right|_{x=0}\left(=v_{-1} \mathbf{1}\right)=v ;
$$

and for $u, v \in V$,

$$
\begin{aligned}
& x_{0}^{-1} \delta\left(\frac{x_{1}-x_{2}}{x_{0}}\right) Y\left(u, x_{1}\right) Y\left(v, x_{2}\right)-x_{0}^{-1} \delta\left(\frac{x_{2}-x_{1}}{-x_{0}}\right) Y\left(v, x_{2}\right) Y\left(u, x_{1}\right) \\
= & x_{2}^{-1} \delta\left(\frac{x_{1}-x_{0}}{x_{2}}\right) Y\left(Y\left(u, x_{0}\right) v, x_{2}\right)
\end{aligned}
$$

(the Jacobi identity). 
By taking $\operatorname{Res}_{x_{0}}$ and $\operatorname{Res}_{x_{1}}$ respectively from the Jacobi identity we get Borcherds' commutator formula and iterate formula

$$
\begin{aligned}
& {\left[Y\left(u, x_{1}\right), Y\left(v, x_{2}\right)\right]=\operatorname{Res}_{x_{0}} x_{2}^{-1} \delta\left(\frac{x_{1}-x_{0}}{x_{2}}\right) Y\left(Y\left(u, x_{0}\right) v, x_{2}\right) } \\
& Y\left(Y\left(u, x_{0}\right) v, x_{2}\right) \\
= & \operatorname{Res}_{x_{1}}\left(x_{0}^{-1} \delta\left(\frac{x_{1}-x_{2}}{x_{0}}\right) Y\left(u, x_{1}\right) Y\left(v, x_{2}\right)-x_{0}^{-1} \delta\left(\frac{x_{2}-x_{1}}{-x_{0}}\right) Y\left(v, x_{2}\right) Y\left(u, x_{1}\right)\right)(2 .
\end{aligned}
$$

In terms of components, we have

$$
\begin{aligned}
& {\left[u_{m}, v_{n}\right]=\sum_{i \geq 0}\left(\begin{array}{c}
m \\
i
\end{array}\right)\left(u_{i} v\right)_{m+n-i},} \\
& \left(u_{m} v\right)_{n}=\sum_{i \geq 0}\left(\begin{array}{c}
m \\
i
\end{array}\right)(-1)^{i}\left(u_{m-i} v_{n+i}-(-1)^{m} v_{m+n-i} u_{i}\right)
\end{aligned}
$$

for $m, n \in \mathbb{Z}$. Let $\mathcal{D}$ be the linear operator on $V$ defined by

$$
\mathcal{D}(v)=v_{-2} \mathbf{1}\left(=\left.\left(\frac{d}{d x} Y(v, x) \mathbf{1}\right)\right|_{x=0}\right) .
$$

Then (cf. [LL])

$$
\begin{aligned}
& {[\mathcal{D}, Y(u, x)]=Y(\mathcal{D} u, x)=\frac{d}{d x} Y(u, x),} \\
& Y(u, x) v=e^{x \mathcal{D}} Y(v,-x) u \quad \text { for } u, v \in V .
\end{aligned}
$$

In terms of components, we have

$$
\begin{aligned}
& {\left[\mathcal{D}, u_{m}\right]=(\mathcal{D} u)_{m}=-m u_{m-1}} \\
& u_{m} v=\sum_{i \geq 0}(-1)^{m+i-1} \frac{1}{i !} \mathcal{D}^{i} v_{m+i} u
\end{aligned}
$$

for $u, v \in V, m \in \mathbb{Z}$.

It follows from Borcherds' commutator formula (2.13) that all the operators $u_{m}$ on $V$, for $u \in V, m \in \mathbb{Z}$, linearly span a Lie subalgebra $\mathfrak{g}_{V}$ of $\mathfrak{g l}(V)$ (the general linear Lie algebra). On the other hand, form the vector space

$$
L(V)=V \otimes \mathbb{C}\left[t, t^{-1}\right]
$$

and define a bilinear multiplication on $L(V)$ by

$$
\left[u \otimes t^{m}, v \otimes t^{n}\right]=\sum_{i \geq 0}\left(\begin{array}{c}
m \\
i
\end{array}\right)\left(u_{i} v \otimes t^{m+n-i}\right)
$$


for $u, v \in V, m, n \in \mathbb{Z}$. It was proved (see [FFR], [Li1], [MP]) that the multiplication on $L(V)$ reduces to a Lie algebra structure on the quotient space

$$
\mathcal{L}(V)=L(V) / D L(V),
$$

where $D=\mathcal{D} \otimes 1+1 \otimes \frac{d}{d t}$. The Lie algebra $\mathfrak{g}_{V}$ is naturally a homomorphic image of $\mathcal{L}(V)$. Notice that the Lie algebra structures on $\mathfrak{g}_{V}$ and $\mathcal{L}(V)$ only use the singular part of $Y(u, x) v$ for $u, v \in V$. This essentially motivated the introduction of the notions of conformal algebra and vertex Lie algebra. Following [FB] we use conformal algebra and vertex Lie algebra synonymously in this paper.

The following notion of vertex Lie algebra is due to $[\mathrm{K}]$ under the name of conformal algebra and independently due to $[\mathrm{P}]$ (see Remark 2.6):

Definition 2.3 A vertex Lie algebra is a vector space $A$ equipped with a linear operator $\partial$ and equipped with $\mathbb{C}$-bilinear products $(a, b) \mapsto a_{n} b$ for $a, b \in A, n \in \mathbb{N}$, such that the following axioms hold for $a, b, c \in A, m, n \in \mathbb{N}$ :

(C0) $a_{n} b=0$ for $n$ sufficiently large;

(C1) $(\partial a)_{n} b=-n a_{n-1} b$;

$(\mathrm{C} 2)$

$$
a_{n} b=\sum_{i \geq 0}(-1)^{n+i+1} \frac{1}{i !} \partial^{i} b_{n+i} a ;
$$

(C3)

$$
a_{m} b_{n} c-b_{n} a_{m} c=\sum_{i=0}^{m}\left(\begin{array}{c}
m \\
i
\end{array}\right)\left(a_{i} b\right)_{m+n-i} c .
$$

Remark 2.4 In view of (2.18), (2.19) and (2.13), any vertex algebra $V$ is naturally a vertex Lie algebra with $\partial=\mathcal{D}$, the operator defined in (2.15).

In the notion of vertex Lie algebra, the $\mathbb{C}$-bilinear products (in terms of generating functions) and the axiom ( $\mathrm{C} 0$ ) amount to a linear map

$$
\begin{aligned}
Y_{-}: A & \rightarrow \operatorname{Hom}\left(A, x^{-1} A\left[x^{-1}\right]\right) \\
a & \mapsto Y_{-}(a, x)=\sum_{n \geq 0} a_{n} x^{-n-1} \quad\left(\text { where } a_{n} \in \text { End } A\right) .
\end{aligned}
$$

With the axiom $(\mathrm{C} 0)$ being included in the map $Y_{-}$, the axioms $(\mathrm{C} 1)-(\mathrm{C} 3)$ amount to

$$
\begin{aligned}
& Y_{-}(\partial a, x)=\frac{d}{d x} Y_{-}(a, x) \\
& Y_{-}(a, x) b=\operatorname{Sing}\left(e^{x \partial} Y_{-}(b,-x) a\right) \\
& {\left[Y_{-}\left(a, x_{1}\right), Y_{-}\left(b, x_{2}\right)\right]=\operatorname{Sing}\left(\sum_{i \geq 0}\left(x_{1}-x_{2}\right)^{-i-1} Y_{-}\left(a_{i} b, x_{2}\right)\right)} \\
& =\operatorname{Sing}\left(Y_{-}\left(Y_{-}\left(a, x_{1}-x_{2}\right) b, x_{2}\right)\right) .
\end{aligned}
$$


Differentiating both sides of (2.26) and then using the properties (2.26) and (2.25) we get

$$
\begin{aligned}
\frac{d}{d x} Y_{-}(a, x) b & =\operatorname{Sing}\left(\partial e^{x \partial} Y_{-}(b,-x) a-e^{x \partial} Y_{-}(\partial b,-x) a\right) \\
& =\partial Y_{-}(a, x) b-Y_{-}(a, x) \partial b .
\end{aligned}
$$

Thus (see $[K])$

$$
\left[\partial, Y_{-}(a, x)\right]=\frac{d}{d x} Y_{-}(a, x)=Y_{-}(\partial a, x) \quad \text { for } a \in A
$$

Equivalently we have

$$
e^{x_{1} \partial} Y_{-}(a, x) e^{-x_{1} \partial}=e^{x_{1} \frac{d}{d x}} Y_{-}(a, x)=Y_{-}\left(e^{x_{1} \partial} a, x\right)=Y_{-}\left(a, x+x_{1}\right)
$$

for $a \in A$. Following $[\mathrm{P}]$, we call (2.28) the half commutator formula and call the following identity the half Jacobi identity:

$$
\begin{aligned}
& \operatorname{Sing}\left(x_{0}^{-1} \delta\left(\frac{x_{1}-x_{2}}{x_{0}}\right) Y_{-}\left(a, x_{1}\right) Y_{-}\left(b, x_{2}\right)-x_{0}^{-1} \delta\left(\frac{x_{2}-x_{1}}{-x_{0}}\right) Y_{-}\left(b, x_{2}\right) Y_{-}\left(a, x_{1}\right)\right) \\
= & \operatorname{Sing}\left(x_{2}^{-1} \delta\left(\frac{x_{1}-x_{0}}{x_{2}}\right) Y_{-}\left(Y_{-}\left(a, x_{0}\right) b, x_{2}\right)\right) .
\end{aligned}
$$

We refer to (2.31) applied to a vector $c \in A$ as the half Jacobi identity for the triple $(a, b, c)$ and similarly we refer to (2.28) applied to a vector $c \in A$ as the half commutator formula for the triple $(a, b, c)$.

The following result is due to Primc ([P], Lemma 6.1; notice that the $D$-operator in the setting of Lemma 6.1 was not used in the proof):

Proposition 2.5 Let $A$ be a vector space equipped with a linear map $Y_{-}$from $A$ to $\operatorname{Hom}\left(A, x^{-1} A\left[x^{-1}\right]\right)$. Then the half commutator relation (2.28) is equivalent to the half Jacobi identity relation (2.31).

Remark 2.6 In $[\mathrm{P}]$, a vertex Lie algebra was defined to be a vector space $A$ equipped with a linear operator $D$ and equipped with a linear map $Y_{-}$from $A$ to $\operatorname{Hom}\left(A, x^{-1} A\left[x^{-1}\right]\right)$ such that $(2.26),(2.29)$ with $D$ in place of $\partial$ and (2.31) hold. In view of Proposition 2.5, this definition is equivalent to Definition 2.3. We often denote a vertex Lie algebra by $\left(A, Y_{-}, \partial\right)$ and refer to $\left(Y_{-}, \partial\right)$ as the vertex Lie algebra structure.

Now we discuss a certain symmetry of the half skew symmetry and the half Jacobi identity, which will be useful in constructing vertex Lie (Poisson) algebras.

Lemma 2.7 Let $V$ be a vector space equipped with a linear operator $\partial$ and let $A(x), B(x) \in$ $x^{-1} V\left[x^{-1}\right]$. Then

$$
A(x)=\operatorname{Sing}\left(e^{x \partial} B(-x)\right)
$$

if and only if

$$
B(x)=\operatorname{Sing}\left(e^{x \partial} A(-x)\right)
$$


Proof. From the obvious symmetry we only need to prove that (2.32) implies (2.33). Using (2.32) and Lemma 2.1 we obtain (2.33) as

$$
\operatorname{Sing}\left(e^{x \partial} A(-x)\right)=\operatorname{Sing}\left(e^{x \partial} \operatorname{Sing}\left(e^{-x \partial} B(x)\right)\right)=\operatorname{Sing} B(x)=B(x) .
$$

The following is a simple observation from the $S_{3}$-symmetry of the Jacobi identity for vertex algebras (see [FHL]):

Proposition 2.8 Let $A$ be a vector space equipped with a linear operator $\partial$ and equipped with a linear map

$$
\begin{aligned}
Y_{-}: A & \rightarrow \operatorname{Hom}\left(A, x^{-1} A\left[x^{-1}\right]\right) \\
a & \mapsto Y_{-}(a, x)
\end{aligned}
$$

such that the following conditions hold for all $a, b \in A$ :

$$
\begin{aligned}
& {\left[\partial, Y_{-}(a, x)\right]=\frac{d}{d x} Y_{-}(a, x)} \\
& Y_{-}(a, x) b=\operatorname{Sing}\left(e^{x \partial} Y_{-}(b,-x) a\right) .
\end{aligned}
$$

Then the half Jacobi identity for an ordered triple $(a, b, c)$ implies the half Jacobi identity for any permutation of $(a, b, c)$.

Proof. Notice that from (2.36) we have the following conjugation formula

$$
e^{x_{1} \partial} Y_{-}(a, x) e^{-x_{1} \partial}=Y_{-}\left(a, x+x_{1}\right) \quad \text { for } a \in A \text {. }
$$

Then using the same argument of [FHL] (and using Lemma 2.1, the half skew symmetry and the conjugation formula) we have the $S_{3}$-symmetry of the half Jacobi identity.

Combining Propositions 2.5 and 2.8 we immediately have:

Proposition 2.9 Let $A, \partial$ and $Y_{-}$be as in Proposition 2.8 and let $a, b, c \in A$. Then the half commutator formula for $(a, b, c)$ implies the half commutator formula for any permutation of $(a, b, c)$.

The following result, which is due to Primc $[\mathrm{P}]$, relates a vertex Lie algebra canonically with an honest Lie algebra:

Proposition 2.10 Let $A$ be a vector space equipped with a linear operator $\partial$ and equipped with a linear map $Y_{-}$from $A$ to $\operatorname{Hom}\left(A, x^{-1} A\left[x^{-1}\right]\right)$, such that

$$
\left[\partial, Y_{-}(a, x)\right]=Y_{-}(\partial a, x)=\frac{d}{d x} Y_{-}(a, x) \quad \text { for } a \in A
$$

Set

$$
L(A)=A \otimes \mathbb{C}\left[t, t^{-1}\right]
$$


and set

$$
\hat{\partial}=\partial \otimes 1+1 \otimes \frac{d}{d t} \in \text { End } L(A)
$$

Define

$$
\left[a \otimes t^{m}, b \otimes t^{n}\right]=\sum_{i \geq 0}\left(\begin{array}{c}
m \\
i
\end{array}\right) a_{i} b \otimes t^{m+n-i}
$$

for $a, b \in A, m, n \in \mathbb{N}$, where $Y_{-}(a, x) b=\sum_{n \geq 0} a_{n} b x^{-n-1}$. Then $A$ is a vertex Lie algebra if and only if $L(A) / \hat{\partial} L(A)$ is a Lie algebra.

Let $R$ be a vertex Lie algebra. Set

$$
\mathcal{L}(R)=L(R) / \hat{\partial} L(R),
$$

the Lie algebra associated to $R$ through Proposition 2.10. Denote by $\rho$ the quotient map from $L(R)$ to $\mathcal{L}(R)$. Then $\operatorname{ker} \rho=\hat{\partial} L(R)$.

Remark 2.11 A different notion of vertex Lie algebra was formulated in [DLM2] with the Lie algebra structure and the surjective map $\rho$ as the main components.

Remark 2.12 It is straightforward to see that a vertex Lie algebra homomorphism from $R_{1}$ to $R_{2}$ naturally gives rise to a Lie algebra homomorphism from $\mathcal{L}\left(R_{1}\right)$ to $\mathcal{L}\left(R_{2}\right)$. Then we have a functor from the category of vertex Lie algebras to the category of Lie algebras.

Let $R$ be a vertex Lie algebra. Then we have a canonical polar decomposition of the associated Lie algebra (see [DLM2], $[\mathrm{P}])$ :

$$
\mathcal{L}(R)=\mathcal{L}(R)_{+} \oplus \mathcal{L}(R)_{-},
$$

where

$$
\mathcal{L}(R)_{-}=\rho\left(R \otimes t^{-1} \mathbb{C}\left[t^{-1}\right]\right) \quad \text { and } \quad \mathcal{L}(R)_{+}=\rho(R \otimes \mathbb{C}[t])
$$

are subalgebras. For $a \in R, n \in \mathbb{Z}$, set

$$
a(n)=\rho\left(a \otimes t^{n}\right) \in \mathcal{L}(R) .
$$

Remark 2.13 For any $\mathcal{L}(R)$-module $W$, we also use $a(n)$ for the corresponding operator on $W$. It is clear that $R$ is a natural $\mathcal{L}(R)_{+}$-module with $a(n)$ acting as $a_{n}$ for $a \in R, n \geq$ 0 .

Remark 2.14 Let $R$ be a vertex Lie algebra. Then $R / \partial R$ is a Lie algebra with

$$
[a+\partial R, b+\partial R]=a_{0} b+\partial R
$$

for $a, b \in R$ (see [B1]). On the other hand, it is easy to see that $\rho(R)$ is a Lie subalgebra of $\mathcal{L}(R)_{+}$and ker $\rho \cap R=\partial R$. Then $R / \partial R$ is isomorphic to the Lie subalgebra $\rho(R)$ of $\mathcal{L}(R)_{+}$. We denote the Lie algebra $R / \partial R=\rho(R)$ by $\mathcal{L}(R)_{0}$. 
An $R$-module (see $[\mathrm{K}])$ is an $\mathcal{L}(R)_{+}$-module $W$ with an action of $\partial$ such that

$$
\left[\partial, Y_{-}(u, x)\right]=\frac{d}{d x} Y_{-}(\partial u, x) \quad \text { for } u \in R .
$$

View $\mathbb{C}$ as a trivial $\mathcal{L}(R)_{+}$-module and form the induced module

$$
\mathcal{V}(R)=U(\mathcal{L}(R)) \otimes_{U\left(\mathcal{L}(R)_{+}\right.} \mathbb{C} .
$$

In view of the Poincaré-Birkhoff-Witt theorem, we have

$$
\mathcal{V}(R)=U\left(\mathcal{L}(R)_{-}\right),
$$

as a vector space, so that we may and do consider $\mathbb{C}$ as a subspace of $\mathcal{V}(R)$. Set

$$
1=1 \otimes 1 \in \mathcal{V}(R) .
$$

For the same reason, we consider $R$ as a subspace of $\mathcal{V}(R)$ through the map $a \mapsto a(-1) \mathbf{1}$.

The following result was proved in $[\mathrm{DLM} 2]$ and $[\mathrm{P}]$ (see also $[\mathrm{FB}]$ and $[\mathrm{K}]$ ):

Theorem 2.15 There exists a unique vertex algebra structure on the $\mathcal{L}(R)$-module $\mathcal{V}(R)$ such that $\mathbf{1}$ is the vacuum vector and such that

$$
Y(a, x)=\sum_{n \in \mathbb{Z}} a(n) x^{-n-1} \quad \text { for } a \in R .
$$

The subspace $R$ generates $\mathcal{L}(R)$ as a vertex algebra. Furthermore, any restricted $\mathcal{L}(R)$ module $W$, in the sense that for any $w \in W$ and $a \in R, a(n) w=0$ for $n$ sufficiently large, is a natural $\mathcal{V}(R)$-module.

The following result is due to Primc in $[\mathrm{P}]$ :

Theorem 2.16 Let $R$ be a vertex Lie algebra and let $\mathcal{V}(R)$ be the vertex algebra associated with $R$. Then the identification map of $R$ as a subspace of $\mathcal{V}(R)$ is a vertex Lie algebra homomorphism. Furthermore, for any vertex algebra $V$ and for any vertex Lie algebra homomorphism $f$ from $R$ to $V$ viewed as a vertex Lie algebra, there exists a unique vertex algebra homomorphism $\bar{f}$ from $\mathcal{V}(R)$ to $V$, extending $f$.

The following result is due to Primc [P] (it was also proved in [DLM2] for $R=V$ a vertex algebra):

Proposition 2.17 Let $R$ be a vertex Lie algebra. Then the linear map

$$
\begin{aligned}
R & \rightarrow \mathcal{L}(R)_{-} \\
a & \mapsto a(-1)
\end{aligned}
$$

is a bijection. 
Since $R$ is an $\mathcal{L}(R)_{+-}$-module, the symmetric algebra $S(R)$ is naturally an $\mathcal{L}(R)_{+^{-}}$ module. On the other hand, $\mathcal{V}(R)$ is also an $\mathcal{L}(R)_{+}$-module. In particular, both $S(R)$ and $\mathcal{V}(R)$ are natural $\mathcal{L}(R)_{0}$-modules. The following is an analogue of a classical result in Lie theory (cf. [Di]):

Proposition 2.18 Let $R$ be a vertex Lie algebra. Then the linear map $\omega$ from $S(R)$ to $\mathcal{V}(R)$, defined by

$$
\omega\left(u^{(1)} \cdots u^{(r)}\right)=\frac{1}{r !} \sum_{\sigma \in S_{r}} u^{(\sigma(1))}(-1) \cdots u^{(\sigma(r))}(-1) \mathbf{1}
$$

for $r \geq 0, u^{(i)} \in R$, is an $\mathcal{L}(R)_{0}$-module isomorphism, where $S_{r}$ is the symmetric group on $\{1, \ldots, r\}$.

Proof. In view of Proposition 2.17 and the Poincaré-Birkhoff-Witt theorem, we have a linear isomorphism

$$
\begin{aligned}
f: U\left(\mathcal{L}(R)_{-}\right) & \rightarrow \mathcal{V}(R) \\
u^{(1)}(-1) \cdots u^{(r)}(-1) & \mapsto u^{(1)}(-1) \cdots u^{(r)}(-1) \mathbf{1}
\end{aligned}
$$

for $r \geq 0, u^{(i)} \in R$. View $R$ as a Lie algebra with the transported structure from

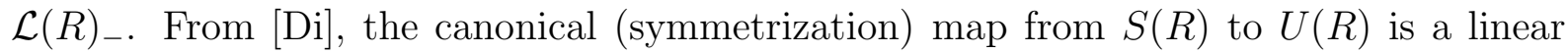
isomorphism. Consequently, the linear map $\omega$ is a linear isomorphism.

Furthermore, for $u, v \in R, w \in \mathcal{V}(R)$, from Borcherds' commutator formula we have

$$
v(0) u(-1) w=u(-1) v(0) w+\left(v_{0} u\right)(-1) w .
$$

Since $v(0) \mathbf{1}=0$, it follows that $\omega$ is an $\mathcal{L}(R)_{0}$-module homomorphism. Therefore, $\omega$ is an $\mathcal{L}(R)_{0}$-module isomorphism.

For a module $W$ for a Lie algebra $\mathfrak{g}$, we set

$$
W^{\mathfrak{g}}=\{w \in W \mid \mathfrak{g} w=0\} .
$$

As an immediate consequence of Proposition 2.18 we have:

Corollary 2.19 Let $\mathfrak{g}$ be a Lie subalgebra of $\mathcal{L}(R)_{0}$. Then the linear map $\omega$ defined in Proposition 2.18 gives rise to a linear isomorphism from $S(R)^{\mathfrak{g}}$ onto $\mathcal{V}(R)^{\mathfrak{g}}$.

Remark 2.20 A derivation (see [Lia], $[\mathrm{K}]$ ) of a vertex algebra $V$ is a linear endomorphism map $f$ of $V$ such that

$$
f(Y(u, x) v)=Y(f(u), x) v+Y(u, x) f(v) \quad \text { for } u, v \in V .
$$

For any $u \in V, u_{0}$ is a derivation of $V$. All derivations of $V$ form a Lie subalgebra Der $V$ of $\mathfrak{g r}(V)$ and all derivations $u_{0}$ for $u \in V$ form an ideal of Der $V$, which is a quotient algebra of the Lie algebra $\mathcal{L}(V)_{0}$. For any Lie subalgebra $\mathfrak{g}$ of Der $V$, it is straightforward to show (cf. $[\mathrm{K}]$ ) that $V^{\mathfrak{g}}$ is a vertex subalgebra of $V$. 
Motivated by the Virasoro algebra and affine Lie algebras, following [DLM2] we next consider certain quotient vertex algebras of $\mathcal{V}(R)$. First we have (cf. [DLM2]):

Lemma 2.21 Let $R$ be a vertex Lie algebra and let $a \in R$ be such that $\partial a=0$. Then

$$
\begin{aligned}
& Y_{-}(a, x) u=0=Y_{-}(u, x) a \quad \text { for all } u \in R \\
& a(n)=0 \quad \text { for } n \neq-1 .
\end{aligned}
$$

Furthermore, a lies in the center of $\mathcal{V}(R)$ and $a(-1)$ is a central element of $\mathcal{L}(R)$.

Proof. With $\partial a=0$, we have

$$
\frac{d}{d x} Y_{-}(a, x)=Y_{-}(\partial a, x)=0
$$

which immediately implies that $Y_{-}(a, x)=0$ because $Y_{-}(a, x) \in \operatorname{Hom}\left(R, x^{-1} R\left[x^{-1}\right]\right)$. It follows from the half skew symmetry that $Y_{-}(u, x) a=0$ for $u \in R$.

With $a_{i} b=0$ for $i \geq 0, b \in R$, it follows from the commutator formula that $a(n)$ is a central element of $\mathcal{L}(R)$ for any $n \in \mathbb{Z}$. We also have

$$
a(n)=\rho\left(a \otimes t^{n}\right)=\frac{1}{n+1} \rho\left(\hat{\partial}\left(a \otimes t^{n+1}\right)\right)=0 \quad \text { for } n \neq-1,
$$

as elements of $\mathcal{L}(R)$. Consequently,

$$
a(n)=0 \quad \text { for } n \neq-1,
$$

as operators on $\mathcal{V}(R)$. It follows from the commutator formula that $a$ is in the center of $\mathcal{V}(R)$.

For a vector space $U$, by a partially defined linear functional on $U$ we mean a linear functional on some subspace of $U$. Rigorously speaking, a partially defined linear functional on $U$ consists of a linear subspace $D_{\lambda}$ of $U$ and a linear functional $\lambda$ on $D_{\lambda}$.

Let $\lambda$ be a partially defined linear functional on ker $\partial$ with domain $D_{\lambda}$. Denote by $I_{\lambda}$ the $\mathcal{L}(R)$-submodule of $\mathcal{V}(R)$ generated by the vectors $a-\lambda(a)$ for $a \in D_{\lambda} \subset$ ker $\partial$. From Lemma 2.21 we have

$$
\mathcal{D}(a-\lambda(a))=\mathcal{D} a=a_{-2} \mathbf{1}=a(-2) \mathbf{1}=0 \quad \text { for } a \in D_{\lambda} \subset \operatorname{ker} \partial,
$$

where $\mathcal{D}$ is the $\mathcal{D}$-operator of $\mathcal{V}(R)$. It follows that $I_{\lambda}$ is $\mathcal{D}$-stable. Since $R$ generates $\mathcal{V}(R)$ as a vertex algebra, $I_{\lambda}$ is an ideal of $\mathcal{V}(R)$ (cf. [LL]). Following [DLM2] we set

$$
\mathcal{V}_{\lambda}(R)=\mathcal{V}(R) / I_{\lambda}
$$

a vertex algebra. Note that $\mathcal{V}_{\lambda}(R)$ is still an $\mathcal{L}(R)$-module. We also have

$$
\mathcal{V}_{\lambda}(R)=U(\mathcal{L}(R)) \otimes_{U\left(\mathcal{L}(R)_{+} \oplus D_{\lambda}\right)} \mathbb{C}_{\lambda}
$$

as an $\mathcal{L}(R)$-module, where $\mathbb{C}_{\lambda}=\mathbb{C}$ on which $\mathcal{L}(R)_{+}$acts as zero and $a$ acts as $\lambda(a)$ for $a \in D_{\lambda} \subset$ ker $\partial$, where $D_{\lambda}$ is considered as a (central) subalgebra of $\mathcal{L}(R)_{-}$through the map $a \mapsto a(-1)$ (recall Lemma 2.21). 
Remark 2.22 Let $\lambda$ be the partially defined linear functional on $\operatorname{ker}_{R} \partial$ with $D_{\lambda}=0$. Then we easily see that $\mathcal{V}_{\lambda}(R)=\mathcal{V}(R)$.

Remark 2.23 Let $R$ be a vertex algebra, let $\lambda$ be a partially defined linear functional on $\operatorname{ker}_{R} \partial$ and let $\mathcal{V}_{\lambda}(R)$ be the vertex algebra associated with $R$ and $\lambda$. Then it follows immediately from Primc's result (Theorem 2.16) that for any vertex algebra $V$ and for any vertex Lie algebra homomorphism $f$ from $R$ to $V$ viewed as a vertex Lie algebra, such that

$$
f(a)=\lambda(a) \mathbf{1} \quad \text { for } a \in D_{\lambda} \subset \operatorname{ker}_{R} \partial,
$$

there exists a unique vertex algebra homomorphism $\bar{f}$ from $\mathcal{V}_{\lambda}(R)$ to $V$ such that $\bar{f} \psi$ extends $f$, where $\psi$ is the quotient map from $\mathcal{V}(R)$ onto $\mathcal{V}_{\lambda}(R)$.

On the other hand, denote by $J_{\lambda}$ the ideal of the symmetric algebra $S(R)$, generated by the vectors $a-\lambda(a)$ for $a \in D_{\lambda} \subset \operatorname{ker} \partial$. Set

$$
S_{\lambda}(R)=S(R) / J_{\lambda},
$$

the quotient algebra. Since from Lemma $2.21 u(i)(a-\lambda(a))=0$ for $u \in R, i \geq 0, a \in$ $D_{\lambda} \subset \operatorname{ker} \partial, J_{\lambda}$ is an $\mathcal{L}(R)_{+}$-submodule of $S(R)$, so that $S_{\lambda}(R)$ is also an $\mathcal{L}(R)_{+}$-module.

Proposition 2.24 Let $R$ be a vertex Lie algebra and let $\lambda$ be a partially defined linear functional on $\operatorname{ker}_{R} \partial$. Then the linear map $\omega$ defined in Proposition 2.18 reduces to an $\mathcal{L}(R)_{0}$-isomorphism $\omega_{\lambda}$ from $S_{\lambda}(R)$ onto $\mathcal{V}_{\lambda}(R)$. Furthermore, for any Lie subalgebra $\mathfrak{g}$ of $\mathcal{L}(R)_{0}$, the map $\omega$ reduces to a linear isomorphism from $S_{\lambda}(R)^{\mathfrak{g}}$ onto $\mathcal{V}_{\lambda}(R)^{\mathfrak{g}}$.

Proof. Let $a \in D_{\lambda} \subset \operatorname{ker} \partial, u^{(1)}, \ldots, u^{(n)} \in R$. Since $\left[a(-1), u^{(i)}(-1)\right]=0$, we have

$$
\begin{aligned}
\omega\left(a u^{(1)} \cdots u^{(n)}\right) & =\frac{1}{n !} \sum_{\sigma \in S_{n}} u^{(\sigma(1))}(-1) \cdots u^{(\sigma(n))}(-1) a(-1) \mathbf{1} \\
& =\frac{1}{n !} \sum_{\sigma \in S_{n}} u^{(1)}(-1) \cdots u^{(\sigma(n))}(-1) a
\end{aligned}
$$

so that

$$
\begin{aligned}
\omega\left((a-\lambda(a)) u^{(1)} \cdots u^{(n)}\right) & =\frac{1}{n !} \sum_{\sigma \in S_{n}} u^{(\sigma(1))}(-1) \cdots u^{(\sigma(n))}(-1)(a-\lambda(a)) \\
& =\left(\frac{1}{n !} \sum_{\sigma \in S_{n}} u^{(\sigma(1))}(-1) \cdots u^{(\sigma(n))}(-1)\right)(a-\lambda(a)) .
\end{aligned}
$$

It follows that $\omega\left(J_{\lambda}\right)=I_{\lambda}$, so $\omega$ gives rise to an $\mathcal{L}(R)_{0}$-module isomorphism $\omega_{\lambda}$ from $S_{\lambda}(R)$ onto $\mathcal{V}_{\lambda}(R)$. The second assertion follows easily.

Remark 2.25 The relationship between $S(R)$ and $\mathcal{V}(R)$ and between $S_{\lambda}(R)$ onto $\mathcal{V}_{\lambda}(R)$ will be further studied in Section 4 in the context of vertex Poisson algebras. 


\section{$3 \quad$ Vertex Poisson algebras}

In this section we recall the notion of vertex Poisson algebra and we give certain general construction theorems of vertex Poisson algebras. We apply the construction theorems to show that the symmetric algebra over a vertex Lie algebra has a natural vertex Poisson algebra structure, which was known to Frenkel and Ben-Zvi.

Throughout this paper, by a differential algebra we mean a commutative associative algebra $A$ with identity 1 equipped with a derivation $\partial$. We also often denote the differential algebra by $(A, \partial)$. We say that a subset $U$ of $A$ generates $A$ as a differential algebra if $\partial^{n} U$ for $n \geq 0$ generate $A$ as an algebra.

The following notion of vertex Poisson algebra is due to [FB] (cf. [DLM2] and [EF]):

Definition 3.1 A vertex Poisson algebra is a differential algebra $(A, \partial)$ equipped with a vertex Lie algebra structure $\left(Y_{-}, \partial\right.$ ) (with the same operator $\partial$ ) such that for $a, b, c \in A$,

$$
Y_{-}(a, x)(b c)=\left(Y_{-}(a, x) b\right) c+b\left(Y_{-}(a, x) c\right) .
$$

In terms of components, (3.1) amounts to

$$
a_{n}(b c)=\left(a_{n} b\right) c+b\left(a_{n} c\right) \quad \text { for } a, b, c \in A, n \geq 0,
$$

where $Y_{-}(a, x)=\sum_{n \geq 0} a_{n} x^{-n-1}$. That is, $a_{n}$, for all $a \in A, n \geq 0$, are derivations of $A$. Thus

$$
Y_{-}(a, x) \in x^{-1}(\operatorname{Der} A)\left[\left[x^{-1}\right]\right] \quad \text { for } a \in A .
$$

This implies that $Y_{-}(a, x) 1=0$, and then $Y_{-}(1, x) a=0$ by half skew symmetry. Thus

$$
Y_{-}(1, x)=0 \text {. }
$$

A vertex algebra $V$ is said to be commutative if $\left[Y\left(u, x_{1}\right), Y\left(v, x_{2}\right)\right]=0$ for all $u, v \in V$. From [FHL], $V$ is commutative if and only if $u_{n} v=0$ for all $u, v \in V, n \geq 0$.

Remark 3.2 It was known (see [B1]; cf. [Li1], Proposition 2.1.6, [FB]) that if $V$ is a commutative vertex algebra, then $V$ is an honest commutative associative algebra with the product defined by

$$
u \cdot v=u_{-1} v \quad \text { for } u, v \in V
$$

and with 1 as the identity element. Furthermore, the $\mathcal{D}$-operator $\mathcal{D}$ of $V$ is a derivation and

$$
Y(u, x) v=\left(e^{x \mathcal{D}} u\right) v \quad \text { for } u, v \in V .
$$

Conversely, for any differential algebra $(A, \partial),(A, Y, 1)$ carries the structure of a commutative vertex algebra where $Y$ is defined by $Y(a, x) b=\left(e^{x \partial} a\right) b$ for $a, b \in A$ (see [B1]). This gives rise to a canonical isomorphism between the category of commutative vertex algebras and the category of differential algebras. In view of this, a vertex Poisson algebra structure on a vector space $A$ consists of a commutative vertex algebra structure and a vertex Lie algebra structure with a compatibility condition (see [FB]). 
A $\mathbb{Z}$-graded vertex Poisson algebra is a vertex Poisson algebra $A$ equipped with a $\mathbb{Z}$ grading $A=\bigsqcup_{n \in \mathbb{Z}} A_{(n)}$ such that $A$ as an algebra is $\mathbb{Z}$-graded and such that for $a \in$ $A_{(n)}, n, r \in \mathbb{Z}, m \in \mathbb{N}$,

$$
\begin{aligned}
& \partial A_{(m)} \subset A_{(m+1)}, \\
& a_{m} A_{(r)} \subset A_{(r+n-m-1)} .
\end{aligned}
$$

An ideal of a vertex Poisson algebra $A$ is an ideal $I$ of $A$ as an associative algebra such that

$$
\begin{aligned}
& \partial I \subset I, \\
& a_{n} I \subset I \quad \text { for } a \in A, n \geq 0 .
\end{aligned}
$$

From the half skew symmetry we also have

$$
u_{n} A \subset I \quad \text { for } u \in I, n \geq 0 .
$$

Thus, the quotient space $A / I$ has a natural vertex Poisson algebra structure.

A vertex-Poisson-algebra homomorphism from $A$ to $B$ is an algebra homomorphism $f$ such that

$$
\begin{aligned}
& f \partial=f \partial \\
& f\left(Y_{-}(a, x) b\right)=Y_{-}(f(a), x) f(b) \quad \text { for } a, b \in A .
\end{aligned}
$$

A vertex-Poisson-algebra isomorphism is a bijective vertex-Poisson-algebra homomorphism.

We have the following simple fact:

Lemma 3.3 Let $A$ and $B$ be vertex Poisson algebras and let $f$ be an algebra homomorphism from $A$ to $B$ such that $f \partial=\partial f$. Suppose that

$$
f\left(Y_{-}(u, x) v\right)=Y_{-}(f(u), x) f(v) \quad \text { for } u, v \in U,
$$

where $U$ is a generating subset of $A$ as a differential algebra. Then $f$ is a vertex-Poissonalgebra homomorphism.

Proof. Let $a, b \in A$ be such that

$$
f\left(Y_{-}(a, x) b\right)=Y_{-}(f(a), x) f(b) .
$$

Using the first equality of (2.29) for both $A$ and $B$ and using the assumption $f \partial=\partial f$ we obtain

$$
\begin{aligned}
f\left(Y_{-}(a, x) \partial b\right) & =f\left(\partial Y_{-}(a, x) b\right)-\frac{d}{d x} f\left(Y_{-}(a, x) b\right) \\
& =\partial f\left(Y_{-}(a, x) b\right)-\frac{d}{d x} Y_{-}(f(a), x) f(b) \\
& =\partial Y_{-}(f(a), x) f(b)-\frac{d}{d x} Y_{-}(f(a), x) f(b) \\
& =Y_{-}(f(a), x) \partial f(b) \\
& =Y_{-}(f(a), x) f(\partial b) .
\end{aligned}
$$


It follows from (3.1) and induction that

$$
f\left(Y_{-}(u, x) a\right)=Y_{-}(f(u), x) f(a) \quad \text { for any } u \in U, a \in A .
$$

Using the half skew symmetry and the assumption $\partial f=f \partial$ we get

$$
f\left(Y_{-}(a, x) u\right)=Y_{-}(f(a), x) f(u) \quad \text { for any } u \in U, a \in A .
$$

Using (3.1) and induction again we get

$$
f\left(Y_{-}(a, x) b\right)=Y_{-}(f(a), x) f(b) \quad \text { for any } a, b \in A .
$$

Thus $f$ is a vertex Poisson algebra homomorphism.

In the following we prove certain general results which will be useful for establishing vertex Poisson algebra structures.

Lemma 3.4 Let $A$ be a differential algebra equipped with a linear map $Y_{-}$from $A$ to $x^{-1}($ Der $A)\left[\left[x^{-1}\right]\right]$ such that $Y_{-}(u, x) v \in x^{-1} A\left[x^{-1}\right]$ for $u, v \in A$. Let $a, b, c, d \in A$ be such that the half skew symmetry holds for all the pairs $(a, c),(a, d),(b, c),(b, d),(a b, c)$, $(a b, d),(a, c d)$, and $(b, c d)$. Then the half skew symmetry holds for the pair $(a b, c d)$ :

$$
Y_{-}(a b, x) c d=\operatorname{Sing}\left(e^{x \partial} Y_{-}(c d,-x) a b\right) .
$$

Proof. Using all the assumptions we have

$$
\begin{aligned}
& Y_{-}(a b, x)(c d) \\
= & \left(Y_{-}(a b, x) c\right) d+c Y_{-}(a b, x) d \\
= & \operatorname{Sing}\left(\left(e^{x \partial} Y_{-}(c,-x)(a b)\right) d+c e^{x \partial} Y_{-}(d,-x)(a b)\right) \\
= & \operatorname{Sing}\left(\left(e^{x \partial}\left(Y_{-}(c,-x) a\right) b\right) d+\left(e^{x \partial}\left(a Y_{-}(c,-x) b\right)\right) d\right) \\
& +\operatorname{Sing}\left(c e^{x \partial}\left(Y_{-}(d,-x) a\right) b+c e^{x \partial}\left(a Y_{-}(d,-x) b\right)\right) \\
= & \operatorname{Sing}\left(\left(Y_{-}(a, x) c\right)\left(e^{x \partial} b\right) d+\left(e^{x \partial} a\right)\left(Y_{-}(b, x) c\right) d\right) \\
& +\operatorname{Sing}\left(c\left(Y_{-}(a, x) d\right)\left(e^{x \partial} b\right)+c\left(e^{x \partial} a\right)\left(Y_{-}(b, x) d\right)\right) .
\end{aligned}
$$

Using (3.19) and the symmetry $(a, b, x) \rightarrow(c, d,-x)$ we get

$$
\begin{aligned}
Y_{-}(c d,-x)(a b)= & \operatorname{Sing}\left(\left(e^{-x \partial}\left(Y_{-}(a, x) c\right) d\right) b+\left(e^{-x \partial}\left(c Y_{-}(a, x) d\right)\right) b\right) \\
& +\operatorname{Sing}\left(a e^{-x \partial}\left(Y_{-}(b, x) c\right) d+a e^{-x \partial}\left(c Y_{-}(b, x) d\right)\right) .
\end{aligned}
$$

Then

$$
\begin{aligned}
& \operatorname{Sing}\left(e^{x \partial} Y_{-}(c d,-x)(a b)\right) \\
= & \left.\operatorname{Sing}\left(Y_{-}(a, x) c\right) d\left(e^{x \partial} b\right)+\left(c Y_{-}(a, x) d\right)\left(e^{x \partial} b\right)\right) \\
& +\operatorname{Sing}\left(\left(e^{x \partial} a\right)\left(Y_{-}(b, x) c\right) d+\left(e^{x \partial} a\right)\left(c Y_{-}(b, x) d\right)\right) .
\end{aligned}
$$

Combining (3.20) with (3.22) we get (3.18).

Furthermore, we have: 
Proposition 3.5 Let $A$ be a differential algebra equipped with a linear map $Y_{-}$from A to $x^{-1}(\operatorname{Der} A)\left[\left[x^{-1}\right]\right]$ such that $Y_{-}(u, x) v \in x^{-1} A\left[x^{-1}\right]$ for $u, v \in A$ and such that $Y_{-}(1, x)=0$. Suppose that

$$
Y_{-}(a, x) b=\operatorname{Sing}\left(e^{x \partial} Y_{-}(b,-x) a\right)
$$

for $a \in A, b \in B$, where $B$ is a generating subset of $A$ as an algebra. Then the half skew symmetry holds for all $a, b \in A$.

Proof. Since $Y_{-}(a, x) \in x^{-1}(\operatorname{Der} A)\left[\left[x^{-1}\right]\right]$ for $a \in A$, we have $Y_{-}(a, x) 1=0$. Then $Y_{-}(a, x) 1=\operatorname{Sing}\left(e^{x \partial} Y_{-}(1,-x) a\right)(=0)$ for any $a \in A$. Because $B$ generates $A$ as an algebra, now it suffices to prove that

$$
Y_{-}\left(a^{(1)} \cdots a^{(m)}, x\right)\left(b^{(1)} \cdots b^{(n)}\right)=\operatorname{Sing}\left(e^{x \partial} Y_{-}\left(b^{(1)} \cdots b^{(n)},-x\right)\left(a^{(1)} \cdots a^{(m)}\right)\right)
$$

for any $a^{(1)}, \ldots, a^{(m)}, b^{(1)}, \ldots, b^{(n)} \in B$ with $m, n \geq 1$. This follows immediately from Lemma 3.4 (and induction on $m+n$ ).

Now we have the following basic result:

Theorem 3.6 Let $A$ be a differential algebra equipped with a linear map $Y_{-}$from $A$ to $x^{-1}(\operatorname{Der} A)\left[\left[x^{-1}\right]\right]$ such that $Y_{-}(a, x) b \in x^{-1} A\left[x^{-1}\right]$ for $a, b \in A, Y_{-}(1, x)=0$ and

$$
Y_{-}(\partial a, x)=\frac{d}{d x} Y_{-}(a, x) \quad \text { for } a \in A .
$$

Assume that $B$ is an ordered generating subset of $A$ as an algebra such that for $a \in A, b \in$ $B$,

$$
Y_{-}(a, x) b=\operatorname{Sing}\left(e^{x \partial} Y_{-}(b,-x) a\right)
$$

and such that for $u, v, w \in B$ with $u \leq v \leq w$,

$$
\left[Y_{-}\left(u, x_{1}\right), Y_{-}\left(v, x_{2}\right)\right] w=\operatorname{Sing}\left(Y_{-}\left(Y_{-}\left(u, x_{1}-x_{2}\right) v, x_{2}\right) w\right) .
$$

Then $A$ is a vertex Poisson algebra. The same assertion holds if we only assume that $B$ generates $A$ as a differential algebra and if in addition we assume

$$
\left[\partial, Y_{-}(a, x)\right]=\frac{d}{d x} Y_{-}(a, x) \quad \text { for } a \in A .
$$

Proof. By Proposition 3.5 we have

$$
Y_{-}(a, x) b=\operatorname{Sing}\left(e^{x \partial} Y_{-}(b,-x) a\right) \quad \text { for all } a, b \in A .
$$

Furthermore, using (3.29) and (3.25) we get

$$
\begin{aligned}
\frac{d}{d x} Y_{-}(a, x) b & =\operatorname{Sing}\left(\partial e^{x \partial} Y_{-}(b,-x) a+e^{x \partial} \frac{d}{d x} Y_{-}(b,-x) a\right) \\
& =\operatorname{Sing}\left(\partial e^{x \partial} Y_{-}(b,-x) a-e^{x \partial} Y_{-}(\partial b,-x) a\right) \\
& =\partial Y_{-}(a, x) b-Y_{-}(a, x) \partial b .
\end{aligned}
$$


Thus

$$
\left[\partial, Y_{-}(a, x)\right]=\frac{d}{d x} Y_{-}(a, x) \quad \text { for any } a \in A .
$$

With the property (3.31), in view of Proposition 2.9, the half commutator formula for $(u, v, w)$ implies the half commutator formula for any permutation of $(u, v, w)$. Since $Y_{-}(a, x) \in x^{-1}$ (Der $\left.A\right)\left[\left[x^{-1}\right]\right]$ and $B$ generates $A,(3.27)$ holds for $u, v \in B$ and for any $w \in A$. Again, since $Y_{-}(a, x) \in x^{-1}$ (Der $\left.A\right)\left[\left[x^{-1}\right]\right]$ and $B$ generates $A$, (3.27) holds for $u \in B$ and for any $v, w \in A$. Using this argument again we see that (3.27) holds for any $u, v, w \in A$. This proves that $A$ is a vertex Poisson algebra.

For the second assertion, for $a \in A, b \in B$, using the conjugation formula (2.30) (which follows from (3.25) and (3.28)) we get

$$
\begin{aligned}
Y_{-}\left(e^{x_{1} \partial} b, x\right) a & =e^{x_{1} \frac{d}{d x}} Y_{-}(b, x) a \\
& =e^{x_{1} \frac{d}{d x}} \operatorname{Sing}\left(e^{x \partial} Y_{-}(a,-x) b\right) \\
& =\operatorname{Sing}_{x}\left(e^{\left(x+x_{1}\right) \partial} e^{x_{1} \frac{d}{d x}} Y_{-}(a,-x) b\right) \\
& =\operatorname{Sing}_{x}\left(e^{x \partial} Y_{-}(a,-x) e^{x_{1} \partial} b\right) .
\end{aligned}
$$

Using the conjugation formula, (3.27) and the Taylor theorem we get

$$
\begin{aligned}
& {\left[Y_{-}\left(e^{z_{1} \partial} u, x_{1}\right), Y_{-}\left(e^{z_{2} \partial} v, x_{2}\right)\right] e^{z \partial} w} \\
& =e^{z_{1} \frac{\partial}{\partial x_{1}}} e^{z_{2} \frac{\partial}{\partial x_{2}}}\left[Y_{-}\left(u, x_{1}\right), Y_{-}\left(v, x_{2}\right)\right] e^{z \partial} w \\
& =e^{\left(z_{1}-z\right) \frac{\partial}{\partial x_{1}}} e^{\left(z_{2}-z\right) \frac{\partial}{\partial x_{2}}} e^{z \partial}\left[Y_{-}\left(u, x_{1}\right), Y_{-}\left(v, x_{2}\right)\right] w \\
& =e^{\left(z_{1}-z\right) \frac{\partial}{\partial x_{1}}} e^{\left(z_{2}-z\right) \frac{\partial}{\partial x_{2}}} e^{z \partial} \operatorname{Sing}\left(Y_{-}\left(Y_{-}\left(u, x_{1}-x_{2}\right) v, x_{2}\right) w\right) \\
& =e^{\left(z_{1}-z\right) \frac{\partial}{\partial x_{1}}} e^{\left(z_{2}-z\right) \frac{\partial}{\partial x_{2}}} \operatorname{Sing}_{x_{1}, x_{2}}\left(Y_{-}\left(Y_{-}\left(u, x_{1}-x_{2}\right) v, x_{2}+z\right) e^{z \partial} w\right) \\
& =\operatorname{Sing}_{x_{1}, x_{2}}\left(Y_{-}\left(Y_{-}\left(u, x_{1}-x_{2}+z_{1}-z_{2}\right) v, x_{2}+z_{2}\right) e^{z \partial} w\right) \\
& =\operatorname{Sing}_{x_{1}, x_{2}}\left(Y_{-}\left(Y_{-}\left(e^{\left(z_{1}-z_{2}\right) \partial} u, x_{1}-x_{2}\right) v, x_{2}+z_{2}\right) e^{z \partial} w\right) \\
& =\operatorname{Sing}_{x_{1}, x_{2}}\left(Y_{-}\left(e^{-z_{2} \partial} Y_{-}\left(e^{z_{1} \partial} u, x_{1}-x_{2}\right) e^{z_{2} \partial} v, x_{2}+z_{2}\right) e^{z \partial} w\right) \\
& =\operatorname{Sing}_{x_{1}, x_{2}}\left(Y_{-}\left(Y_{-}\left(e^{z_{1} \partial} u, x_{1}-x_{2}\right) e^{z_{2} \partial} v, x_{2}\right) e^{z \partial} w\right) \text {. }
\end{aligned}
$$

Since $\partial^{n} U$ for $n \geq 0$ generate $A$ as an algebra, now it follows immediately from the first assertion.

It is well known that for any Lie algebra $\mathfrak{g}$, there exists a unique Poisson algebra structure $\{\cdot, \cdot\}$ on the symmetric algebra $S(\mathfrak{g})$ such that $\{u, v\}=[u, v]$ for $u, v \in \mathfrak{g}$. On the other hand, if there is a Poisson algebra structure $\{\cdot, \cdot\}$ on the symmetric algebra $S(U)$ for a vector space $U$ such that $\{u, v\} \in U$ for $u, v \in U$, then $(U,\{\cdot, \cdot\})$ must be a Lie algebra. The following is a vertex analogue of this fact (cf. [FB], Example 15.2.2): 
Proposition 3.7 Let $R$ be a vector space equipped with a linear operator $\partial$ and let $Y_{-}^{0}$ be a linear map from $R$ to $\operatorname{Hom}\left(R, x^{-1} R\left[x^{-1}\right]\right)$. Denote by $S(R)$ the symmetric algebra over $R$ and we extend $\partial$ (uniquely) to a derivation of $S(R)$. Then $Y_{-}^{0}$ extends to a vertex Poisson algebra structure $Y_{-}$on $(S(R), \partial)$ if and only if $\left(R, \partial, Y_{-}^{0}\right)$ carries the structure of a vertex Lie algebra. Furthermore, such an extension is unique.

Proof. The "only if" part is clear. It is also clear that if $Y_{-}^{0}$ extends to a vertex Poisson algebra structure $Y_{-}$on $(S(R), \partial)$, it must be unique. So, we must prove that if $\left(R, \partial, Y_{-}^{0}\right)$ carries the structure of a vertex Lie algebra, then $Y_{-}^{0}$ extends to a vertex Poisson algebra structure $Y_{-}$on $S(R)$. First, for $u \in R$, we define a unique element

$$
\tilde{Y}_{-}^{0}(u, x) \in x^{-1}(\operatorname{Der} S(R))\left[\left[x^{-1}\right]\right]
$$

by

$$
\tilde{Y}_{-}^{0}(u, x) v=Y_{-}^{0}(u, x) v \quad \text { for } u, v \in R .
$$

For $a \in S(R)$, we define

$$
Y_{-}(a, x) \in x^{-1}(\operatorname{Der} S(R))\left[\left[x^{-1}\right]\right]
$$

by

$$
Y_{-}(a, x) u=\operatorname{Sing}\left(e^{x \partial} \tilde{Y}_{-}^{0}(u,-x) a\right) \quad \text { for } u \in R .
$$

For $u, v \in R$, using the half-skew symmetry of the vertex Lie algebra $\left(R, \partial, Y_{-}^{0}\right)$ we have

$$
Y_{-}(v, x) u=\operatorname{Sing}\left(e^{x \partial} \tilde{Y}_{-}^{0}(u,-x) v\right)=\operatorname{Sing}\left(e^{x \partial} Y_{-}^{0}(u,-x) v\right)=Y_{-}^{0}(v, x) u .
$$

That is, $Y_{-}$as a linear map from $R \otimes R$ to $x^{-1} R\left[x^{-1}\right]$ extends $Y_{-}^{0}$. Since $Y_{-}(v, x), \tilde{Y}_{-}^{0}(v, x) \in$ $x^{-1}(\operatorname{Der} S(R))\left[\left[x^{-1}\right]\right]$ and $Y_{-}(v, x) u=\tilde{Y}_{-}^{0}(v, x) u$ for all $u \in R$, we have

$$
Y_{-}(v, x) a=\tilde{Y}_{-}^{0}(v, x) a \quad \text { for all } a \in S(R) .
$$

Similarly, since $\left[\partial, Y_{-}^{0}(u, x)\right] v=\frac{d}{d x} Y_{-}^{0}(u, x) v$ for $u, v \in R$, it follows that for $u \in R, a \in$ $S(R)$,

$$
\left[\partial, \tilde{Y}_{-}^{0}(u, x)\right] a=\frac{d}{d x} \tilde{Y}_{-}^{0}(u, x) a .
$$

Then for $a \in S(R), u \in R$, we have

$$
\begin{aligned}
Y_{-}(\partial a, x) u & =\operatorname{Sing}\left(e^{x \partial} \tilde{Y}_{-}^{0}(u,-x) \partial a\right) \\
& =\operatorname{Sing}\left(\partial e^{x \partial} \tilde{Y}_{-}^{0}(u,-x) a+e^{x \partial} \frac{d}{d x} \tilde{Y}_{-}^{0}(\partial u,-x) a\right) \\
& =\frac{d}{d x} \operatorname{Sing}\left(e^{x \partial} \tilde{Y}_{-}^{0}(u,-x) a\right) \\
& =\frac{d}{d x} Y_{-}(a, x) u .
\end{aligned}
$$


Thus

$$
Y_{-}(\partial a, x)=\frac{d}{d x} Y_{-}(a, x)
$$

For $a \in S(R), u \in R$ we also have

$$
Y_{-}(a, x) u=\operatorname{Sing}\left(e^{x \partial} \tilde{Y}_{-}^{0}(u,-x) a\right)=\operatorname{Sing}\left(e^{x \partial} Y_{-}(u,-x) a\right) .
$$

With $\tilde{Y}_{-}^{0}(u, x) v=Y_{-}^{0}(u, x) v$ for $u, v \in R$, it now follows immediately from Theorem 3.6 that $\left(S(R), Y_{-}, \partial\right)$ carries the structure of a vertex Poisson algebra.

Furthermore, we have:

Proposition 3.8 Let $R$ be a vertex Lie algebra and let $\lambda$ be a partially defined linear functional on $\operatorname{ker}_{R} \partial$. Then $J_{\lambda}$, the ideal of $S(R)$ generated by the vectors $a-\lambda(a)$ for $a \in D_{\lambda} \subset \operatorname{ker}_{R} \partial$, is an ideal of the vertex Poisson algebra $S(R)$, so that the quotient space $S(R) / J_{\lambda}$, denoted by $S_{\lambda}(R)$, is naturally a vertex Poisson algebra.

Proof. For $a \in D_{\lambda} \subset \operatorname{ker}_{R} \partial$, we have $\partial(a-\lambda(a))=0$, so that $\partial J_{\lambda} \subset J_{\lambda}$, since $\partial$ is a derivation of $S(R)$. Similarly,

$$
Y_{-}(u, x) J_{\lambda} \subset x^{-1} J_{\lambda}\left[\left[x^{-1}\right]\right] \quad \text { for } u \in S(R),
$$

since $Y_{-}(u, x) \in x^{-1}(\operatorname{Der} S(R))\left[\left[x^{-1}\right]\right]$ and $Y_{-}(u, x)(a-\lambda(a))=0$ for $a \in \operatorname{ker}_{R} \partial$. (Recall Lemma 2.21 that $Y_{-}(u, x) a=0$ for $a \in \operatorname{ker}_{R} \partial$.) Therefore, $J_{\lambda}$ is an ideal of the vertex Poisson algebra $S(R)$.

Just like the Poisson algebra $S(\mathfrak{g})$ associated with a Lie algebra $\mathfrak{g}$, the vertex Poisson algebra $S(R)$ satisfies a certain universal property.

Proposition 3.9 Let $R$ be a vertex Lie algebra and let $S(R)$ be the associated vertex Poisson algebra. Then for any vertex Poisson algebra $A$ and for any vertex Lie algebra homomorphism $g$ from $R$ to $A$ viewed as a vertex Lie algebra, there exists a unique vertex Poisson algebra homomorphism $\bar{g}$ from $S(R)$ to $A$, extending $g$. Furthermore, if

$$
g(a)=\lambda(a) \quad \text { for } a \in D_{\lambda} \subset \operatorname{ker}_{R} \partial,
$$

for some partially defined linear functional $\lambda$ on $\operatorname{ker}_{R} \partial$, the map $\bar{g}$ induces a vertex Poisson algebra homomorphism from $S_{\lambda}(R)$ to $A$.

Proof. The uniqueness is clear, since $R$ generates $S(R)$ as a commutative associative algebra. Let $\bar{g}$ be the unique algebra homomorphism from $S(R)$ to $A$ extending $g$. It remains to show

$$
\begin{aligned}
& \bar{g} \partial(u)=\partial \bar{g}(u) \\
& \bar{g}\left(u_{n} v\right)=\bar{g}(u)_{n} \bar{g}(v) \quad \text { for } u, v \in S(R), n \geq 0 .
\end{aligned}
$$


Let $u, v \in S(R)$ be such that $\bar{g} \partial(u)=\partial \bar{g}(u)$ and $\bar{g} \partial(v)=\partial \bar{g}(v)$. Then

$$
\begin{aligned}
\bar{g}(\partial(u v)) & =\bar{g}(\partial(u)) \bar{g}(v)+\bar{g}(u) \bar{g}(\partial(v)) \\
& =(\partial \bar{g}(u)) \bar{g}(v)+\bar{g}(u) \partial \bar{g}(v) \\
& =\partial \bar{g}(u v) .
\end{aligned}
$$

We also have $\bar{g} \partial(1)=0=\partial \bar{g}(1)$ and

$$
\bar{g} \partial(a)=g \partial a=\partial g(a)=\partial \bar{g}(a) \quad \text { for } a \in R .
$$

Thus $\operatorname{ker}(\bar{g} \partial-\partial \bar{g})$ is a subalgebra of $S(R)$, containing 1 and $R$, so that $\operatorname{ker}(\bar{g} \partial-\partial \bar{g})=$ $S(R)$. This proves (3.45).

Let $a \in R, n \in \mathbb{N}$. We have that $a_{n}$ is a derivation of $S(R), \bar{g}(a)_{n}$ is a derivation of $A$ and that

$$
\begin{aligned}
& \bar{g}\left(a_{n} 1\right)=0=\bar{g}(a)_{n} \bar{g}(1) \\
& \bar{g}\left(a_{n} b\right)=g\left(a_{n} b\right)=g(a)_{n} g(b)=\bar{g}(a)_{n} \bar{g}(b) \quad \text { for } b \in R .
\end{aligned}
$$

The same argument in the proof of (3.45) with $a_{n}$ in place of $\partial$ shows that

$$
\bar{g}\left(a_{n} u\right)=\bar{g}(a)_{n} \bar{g}(u) \quad \text { for } u \in S(R) .
$$

It follows from half skew symmetry and (3.45) that

$$
\bar{g}\left(u_{n} a\right)=\bar{g}(u)_{n} \bar{g}(a) \quad \text { for } u \in S(R), n \in \mathbb{N}, a \in R .
$$

Then (3.46) follows from the same argument of the first paragraph. Therefore, $\bar{g}$ is a vertex Poisson algebra homomorphism. The last assertion is clear.

Just as with the usual Poisson algebra $S(\mathfrak{g})$, we call the vertex Poisson algebra structure on $S(R)$ associated with a vertex Lie algebra $R$ a linear vertex Poisson structure. Next, we shall study "nonlinear" vertex Poisson structures on a free differential algebra.

Let $U$ be a vector space space, fixed for the rest of this section. Set

$$
A=S(\mathbb{C}[\partial] \otimes U),
$$

the symmetric algebra over the space $\mathbb{C}[\partial] \otimes U$, which is a free $\mathbb{C}[\partial]$-module. We shall use the notation $\partial^{i} u$ for $\partial^{i} \otimes u$. Extend $\partial$ (uniquely) to a derivation of $A$. Now, $(A, \partial)$ is a differential algebra. We refer to this differential algebra as the free differential algebra over $U$.

We hope to determine all the vertex Poisson algebra structures on $A$. Clearly, any vertex Poisson algebra structure $Y_{-}$on $A$ is uniquely determined by giving $Y_{-}(u, x) v$ for $u, v \in U$. Furthermore, the half skew-symmetry necessarily holds for any pair $(u, v) \in$ $U \times U$.

We define a weak pre-vertex-Poisson structure on $A$ to be a linear map $Y_{-}^{0}$ from $U \times U$ to $x^{-1} A\left[x^{-1}\right]$ such that

$$
Y_{-}^{0}(u, x) v=\operatorname{Sing}\left(e^{x \partial} Y_{-}^{0}(v,-x) u\right) \quad \text { for } u, v \in U .
$$

The following result, to a certain extent, is analogous to Lemma 7.1 of $[\mathrm{P}]$ : 
Proposition 3.10 Let $Y_{-}^{0}:(u, v) \mapsto Y_{-}^{0}(u, x) v$ be a weak pre-vertex-Poisson structure on A. Then $Y_{-}^{0}$ extends uniquely to a linear map

$$
\begin{aligned}
Y_{-}: A & \rightarrow \operatorname{Hom}\left(A, x^{-1} A\left[x^{-1}\right]\right) \\
a & \mapsto Y_{-}(a, x)=\sum_{n \in \mathbb{N}} a_{n} x^{-n-1}
\end{aligned}
$$

such that for $a, b \in A$,

$$
\begin{aligned}
& Y_{-}(a, x)=\sum_{n \in \mathbb{N}} a_{n} x^{-n-1} \in x^{-1}(\operatorname{Der} A)\left[\left[x^{-1}\right]\right] \\
& {\left[\partial, Y_{-}(a, x)\right]=Y_{-}(\partial a, x)=\frac{d}{d x} Y_{-}(a, x)} \\
& Y_{-}(a, x) b=\operatorname{Sing}\left(e^{x \partial} Y_{-}(b,-x) a\right) .
\end{aligned}
$$

Proof. Suppose that $Y_{-}$is such a linear map. For $u, v \in U$, from (3.57) we have

$$
Y_{-}(u, x) e^{x_{1} \partial} v=e^{x_{1} \partial} e^{-x_{1} \frac{d}{d x}} Y_{-}(u, x) v=e^{x_{1} \partial} e^{-x_{1} \frac{d}{d x}} Y_{-}^{0}(u, x) v
$$

Since $\partial^{m} v$, for $m \in \mathbb{N}, v \in U$, generates $A$ as an algebra and since $Y_{-}(u, x) \in x^{-1}($ Der $A)\left[\left[x^{-1}\right]\right]$, $Y_{-}(u, x) a$ for any $a \in A$ is uniquely determined. Furthermore, using (3.58) and using the same argument we see that such linear map $Y_{-}$is uniquely determined by the properties (3.56)-(3.58).

For the existence of a linear map $Y_{-}$with the required properties, first for $u \in U$ we define

$$
\tilde{Y}_{-}^{0}(u, x) \in x^{-1}(\operatorname{Der} A)\left[\left[x^{-1}\right]\right]
$$

in terms of generating functions by

$$
\tilde{Y}_{-}^{0}(u, x) e^{x_{1} \partial} v=e^{x_{1} \partial} e^{-x_{1} \frac{d}{d x}} Y_{-}^{0}(u, x) v .
$$

Clearly, $\tilde{Y}_{-}^{0}(u, x) \partial^{m} v \in x^{-1} A\left[x^{-1}\right]$, so it follows immediately that $\tilde{Y}_{-}^{0}(u, x) \in \operatorname{Hom}\left(A, x^{-1} A\left[x^{-1}\right]\right)$. It is also clear that $\tilde{Y}_{-}^{0}(u, x) v=Y_{-}^{0}(u, x) v$ for $u, v \in U$.

Differentiating both sides of (3.61) with respect to $x_{1}$ and using (3.61) we get

$$
\begin{aligned}
\tilde{Y}_{-}^{0}(u, x) \partial e^{x_{1} \partial} v & =\partial e^{x_{1} \partial} e^{-x_{1} \frac{d}{d x}} Y_{-}^{0}(u, x) v-e^{x_{1} \partial} \frac{d}{d x} e^{-x_{1} \frac{d}{d x}} Y_{-}^{0}(u, x) v \\
& =\partial \tilde{Y}_{-}^{0}(u, x) e^{x_{1} \partial} v-\frac{d}{d x} \tilde{Y}_{-}^{0}(u, x) e^{x_{1} \partial} v
\end{aligned}
$$

That is,

$$
\left[\partial, \tilde{Y}_{-}^{0}(u, x)\right] e^{x_{1} \partial} v=\frac{d}{d x} \tilde{Y}_{-}^{0}(u, x) e^{x_{1} \partial} v
$$


Because $\partial, \tilde{Y}_{-}^{0}(u, x) \in x^{-1}(\operatorname{Der} A)\left[\left[x^{-1}\right]\right]$, we have

$$
[\partial, \tilde{B}(u, x)]=\frac{d}{d x} \tilde{B}(u, x) \quad \text { for } u \in U .
$$

Now, for $a \in A$, we define

$$
Y_{-}(a, x)=\sum_{n \in \mathbb{N}} a_{n} x^{-n-1} \in x^{-1}(\operatorname{Der} A)\left[\left[x^{-1}\right]\right]
$$

by

$$
Y_{-}(a, x) \partial^{m} u=\operatorname{Sing}\left(e^{x \partial}\left(-\frac{d}{d x}\right)^{m} \tilde{Y}_{-}^{0}(u,-x) a\right)
$$

for $m \in \mathbb{N}, u \in U$. Clearly, $Y_{-}(a, x) \partial^{m} u \in x^{-1} A\left[x^{-1}\right]$. Then

$$
Y_{-}(a, x) b \in x^{-1} A\left[x^{-1}\right] \quad \text { for } a, b \in A \text {. }
$$

For $u, v \in U$, we have

$$
Y_{-}(u, x) v=\operatorname{Sing}\left(e^{x \partial} \tilde{Y}_{-}^{0}(v,-x) u\right)=\operatorname{Sing}\left(e^{x \partial} Y_{-}^{0}(v,-x) u\right)=Y_{-}^{0}(u, x) v .
$$

Hence $Y_{-}$extends $Y_{-}^{0}$.

For $a \in A, u \in U, m \in \mathbb{N}$, we have

$$
\begin{aligned}
& {\left[\partial, Y_{-}(a, x)\right] \partial^{m} u } \\
= & \partial Y_{-}(a, x) \partial^{m} u-Y_{-}(a, x) \partial^{m+1} u \\
= & \operatorname{Sing}\left(\partial e^{x \partial}\left(-\frac{d}{d x}\right)^{m} \tilde{Y}_{-}^{0}(u,-x) a-e^{x \partial}\left(-\frac{d}{d x}\right)^{m+1} \tilde{Y}_{-}^{0}(u,-x) a\right) \\
= & \operatorname{Sing}\left(\frac{d}{d x}\left\{e^{x \partial}\left(-\frac{d}{d x}\right)^{m} \tilde{Y}_{-}^{0}(u,-x) a\right\}\right) \\
= & \frac{d}{d x} \operatorname{Sing}\left(\left\{e^{x \partial}\left(-\frac{d}{d x}\right)^{m} \tilde{Y}_{-}^{0}(u,-x) a\right\}\right) \\
= & \frac{d}{d x} Y_{-}(a, x) \partial^{m} u,
\end{aligned}
$$

and using (3.64) we get

$$
\begin{aligned}
Y_{-}(\partial a, x) \partial^{m} u & =\operatorname{Sing}\left(e^{x \partial}\left(-\frac{d}{d x}\right)^{m} \tilde{Y}_{-}^{0}(u,-x) \partial a\right) \\
& =\operatorname{Sing}\left(e^{x \partial}\left(-\frac{d}{d x}\right)^{m}\left(\partial+\frac{d}{d x}\right) \tilde{Y}_{-}^{0}(u,-x) a\right) \\
& =\frac{d}{d x} \operatorname{Sing}\left(e^{x \partial}\left(-\frac{d}{d x}\right)^{m} \tilde{Y}_{-}^{0}(u,-x) a\right) \\
& =\frac{d}{d x} Y_{-}(a, x) \partial^{m} u .
\end{aligned}
$$


Since

$$
\partial, \quad Y_{-}(a, x), \frac{d}{d x} Y_{-}(a, x), \quad Y_{-}(\partial a, x) \in x^{-1}(\operatorname{Der} A)\left[\left[x^{-1}\right]\right],
$$

we immediately obtain (3.57). For $u, v \in U$, since $Y_{-}(u, x) v=\tilde{Y}_{-}^{0}(u, x) v$, using (3.57) we get

$$
\left(Y_{-}(u, x)-\tilde{Y}_{-}^{0}(u, x)\right) e^{x_{1} \partial} v=e^{x_{1} \partial}\left(Y_{-}\left(u, x-x_{1}\right)-\tilde{Y}_{-}^{0}\left(u, x-x_{1}\right)\right) v=0 .
$$

Thus

$$
Y_{-}(u, x)=\tilde{Y}_{-}^{0}(u, x) \quad \text { on } A .
$$

It now remains to prove the half skew symmetry. First, notice that using (3.57) we get

$$
Y_{-}(a, x) \partial^{m} u=\operatorname{Sing}\left(e^{x \partial}\left(-\frac{d}{d x}\right)^{m} Y_{-}(u,-x) a\right)=\operatorname{Sing}\left(e^{x \partial} Y_{-}\left(\partial^{m} u,-x\right) a\right) .
$$

Then it follows from Proposition 3.5 with $B=\mathbb{C}[\partial] U$ that the half skew symmetry holds for any pair $(a, b)$ of elements of $A$.

The following theorem (which is somewhat analogous to Proposition 7.7 of $[\mathrm{P}]$ ) facilitates the construction of vertex Poisson algebras:

Theorem 3.11 Let $A$ be the free differential algebra over $U$ and let $Y_{-}^{0}$ be a weak prevertex Poisson structure on $A$ such that that for $u, v, w \in U$ from an ordered basis of $U$ with $u \leq v \leq w$,

$$
\begin{aligned}
& \tilde{Y}_{-}^{0}\left(u, x_{1}\right) Y_{-}^{0}\left(v, x_{2}\right) w-\tilde{Y}_{-}^{0}\left(v, x_{2}\right) Y_{-}^{0}\left(u, x_{1}\right) w \\
= & \operatorname{Sing}\left(e^{x_{2} \partial} \tilde{Y}_{-}^{0}\left(w,-x_{2}\right) Y_{-}^{0}\left(u, x_{1}-x_{2}\right) v\right),
\end{aligned}
$$

where $\tilde{Y}_{-}^{0}(u, x) \in x^{-1}(\operatorname{Der} A)\left[\left[x^{-1}\right]\right]$ is uniquely determined by

$$
\tilde{Y}_{-}^{0}(u, x) e^{x_{1} \partial} v=e^{x_{1} \partial} e^{-x_{1} \frac{d}{d x}} Y_{-}^{0}(u, x) v
$$

for $v \in U$. Then $Y_{-}^{0}$ uniquely extends to a vertex Poisson structure $Y_{-}$on $A$.

Proof. The uniqueness follows from Proposition 3.10. Also, from Proposition 3.10, we have a linear map $Y_{-}$from $A$ to $\operatorname{Hom}\left(A, x^{-1} A\left[x^{-1}\right]\right)$ such that $Y_{-}(u, x)=\tilde{Y}_{-}^{0}(u, x)$ for $u \in U$ and such that (3.56)-(3.58) holds. Then for $u, v, w \in U$, from (3.73), using (3.58) we get

$$
\begin{aligned}
& Y_{-}\left(u, x_{1}\right) Y_{-}\left(v, x_{2}\right) w-Y_{-}\left(v, x_{2}\right) Y_{-}\left(u, x_{1}\right) w \\
= & \operatorname{Sing}\left(e^{x_{2} \partial} Y_{-}\left(w,-x_{2}\right) Y_{-}\left(u, x_{1}-x_{2}\right) v\right) \\
= & \operatorname{Sing}\left(Y_{-}\left(Y_{-}\left(u, x_{1}-x_{2}\right) v, x_{2}\right) w\right) .
\end{aligned}
$$

Now, it follows immediately from Theorem 3.6 that $Y_{-}$is a vertex Poisson structure on A. 
Example 3.12 Let $\mathfrak{g}$ be a Lie algebra equipped with a nondegenerate symmetric invariant bilinear form $\langle\cdot, \cdot\rangle$ and let $\ell$ be any complex number. Let $A$ be the free differential algebra over $\mathfrak{g}$. Define

$$
Y_{-}^{0}(u, x) v=[u, v] x^{-1}+\ell\langle u, v\rangle x^{-2} \quad \text { for } u, v \in \mathfrak{g} .
$$

The bilinear map $B$ is a weak pre-vertex Poisson structure on $A$, since

$$
Y_{-}^{0}(u, x) v=[v, u](-x)^{-1}+\ell\langle v, u\rangle(-x)^{-2}=\operatorname{Sing}\left(e^{x \partial} B(v,-x) u\right) .
$$

Since $\tilde{B}(u, x) 1=0$, we have

$$
\begin{aligned}
& \tilde{Y}_{-}^{0}\left(u, x_{1}\right) Y_{-}^{0}\left(v, x_{2}\right) w-\tilde{Y}_{-}^{0}\left(v, x_{2}\right) Y_{-}^{0}\left(u, x_{1}\right) w \\
= & \tilde{Y}_{-}^{0}\left(u, x_{1}\right)\left([v, w] x_{2}^{-1}+\ell\langle v, w\rangle x_{2}^{-2}\right)-\tilde{Y}_{-}^{0}\left(v, x_{2}\right)\left([u, w] x_{1}^{-1}+\ell\langle u, w\rangle x_{1}^{-2}\right) \\
= & Y_{-}^{0}\left(u, x_{1}\right)[v, w] x_{2}^{-1}-Y_{-}^{0}\left(v, x_{2}\right)[u, w] x_{1}^{-1} \\
= & {[u,[v, w]] x_{1}^{-1} x_{2}^{-1}+\ell\langle u,[v, w]\rangle x_{1}^{-2} x_{2}^{-1}-[v,[u, w]] x_{1}^{-1} x_{2}^{-1}-\ell\langle v,[u, w]\rangle x_{2}^{-2} x_{1}^{-1} } \\
= & {[w,[u, v]] x_{1}^{-1} x_{2}^{-1}+\ell\langle w,[u, v]\rangle\left(x_{1}^{-1} x_{2}^{-2}+x_{1}^{-2} x_{2}^{-1}\right) . }
\end{aligned}
$$

On the other hand,

$$
\begin{aligned}
& \operatorname{Sing}\left(e^{x_{2} \partial} \tilde{Y}_{-}^{0}\left(w,-x_{2}\right) Y_{-}^{0}\left(u, x_{1}-x_{2}\right) v\right) \\
= & \operatorname{Sing}\left(e^{x_{2} \partial}\left([w,[u, v]]\left(-x_{2}\right)^{-1}\left(x_{1}-x_{2}\right)^{-1}+\ell\langle w,[u, v]\rangle\left(-x_{2}\right)^{-2}\left(x_{1}-x_{2}\right)^{-1}\right)\right) \\
= & {[w,[u, v]] x_{1}^{-1} x_{2}^{-1}+\ell\langle w,[u, v]\rangle\left(x_{1}^{-1} x_{2}^{-2}+x_{1}^{-2} x_{2}^{-1}\right) . }
\end{aligned}
$$

Thus

$$
\left.\tilde{Y}_{-}^{0}\left(u, x_{1}\right) Y_{-}^{0}\left(v, x_{2}\right) w-\tilde{Y}_{-}^{0}\left(v, x_{2}\right) Y_{-}^{0}\left(u, x_{1}\right) w=\operatorname{Sing}\left(e^{x_{2} \partial} \tilde{Y}_{-}^{0}\left(w,-x_{2}\right) Y_{-}^{0}\left(u, x_{1}-x_{2}\right) v\right\rangle 3.79\right)
$$

for $u, v, w \in \mathfrak{g}$. By Theorem 3.11, $Y_{-}^{0}$ extends uniquely to a vertex Poisson structure on A.

Remark 3.13 From Example 3.12, the subspace $R^{\prime}=\mathbb{C}[\partial] \otimes \mathfrak{g} \oplus \mathbb{C}$ of $A$ is a vertex Lie subalgebra where $\partial \alpha=0, Y_{-}(u, x) \alpha=Y_{-}(\alpha, x) u=0$ and $Y_{-}(u, x) v=[u, v] x^{-1}+$ $\ell\langle u, v\rangle x^{-2}$ for $u, v \in \mathfrak{g}, \alpha \in \mathbb{C}$. It follows that there exists a unique vertex Lie algebra structure on the space $R=\mathbb{C}[\partial] \otimes \mathfrak{g} \oplus \mathbb{C} c$, where $c$ is a symbol, such that $\partial c=0$, $Y_{-}(u, x) c=Y_{-}(c, x) u=0$ and $Y_{-}(u, x) v=[u, v] x^{-1}+c\langle u, v\rangle x^{-2}$ for $u, v \in \mathfrak{g}$. This was proved in $[\mathrm{P}]$ (cf. $[\mathrm{K}]$ ).

\section{Filtered vertex algebras and vertex Poisson alge- bras}

In this section we formulate a notion of filtered vertex algebra and we prove that for any filtered vertex algebra $(V, E)$, the associated graded vector space $\operatorname{gr}_{E} V$ is naturally 
a vertex Poisson algebra. For any vertex algebra $V$, we determine all the filtered vertex algebras $(V, E)$ and we associate a canonical filtered vertex algebra $(V, E)$ to any $\mathbb{N}$-graded vertex algebra $V=\coprod_{n \geq 0} V_{(n)}$ with $V_{(0)}=\mathbb{C} \mathbf{1}$. We also formulate and study a notion of generating subspace with PBW spanning property of a vertex algebra $V$ and we give a connection between this notion and our construction of "good filtration" of $V$.

Definition 4.1 A good filtration of a vertex algebra $V$ is an increasing filtration by subspaces

$$
\cdots \subset E_{-2} \subset E_{-1} \subset E_{0} \subset E_{1} \subset E_{2} \subset \cdots, \quad \cup_{n \in \mathbb{Z}}^{\infty} E_{n}=V,
$$

such that $1 \in E_{0}$ and such that for $u \in E_{m}, v \in E_{n}$,

$$
\begin{aligned}
& u_{j} v \in E_{m+n} \quad \text { for } j<0 \\
& u_{j} v \in E_{m+n-1}\left(\subset E_{m+n}\right) \quad \text { for } j \geq 0 .
\end{aligned}
$$

A filtered vertex algebra is a vertex algebra $V$ equipped with a good filtration $E=\left\{E_{n}\right\}$. We sometimes denote the filtered vertex algebra by $(V, E)$.

As an immediate consequence of definition we have

$$
\mathcal{D} E_{m} \subset E_{m} \quad \text { for } m \in \mathbb{Z}
$$

since $\mathcal{D} v=v_{-2} \mathbf{1} \in E_{m}$ for $v \in E_{m}$ by (4.2) (notice that $\mathbf{1} \in E_{0}$ by assumption).

Let $(V, E)$ be a filtered vertex algebra. Set

$$
\operatorname{gr}_{E} V=\coprod_{n \in \mathbb{Z}}^{\infty}\left(\operatorname{gr}_{E} V\right)_{n}=\coprod_{n \in \mathbb{Z}}^{\infty}\left(E_{n} / E_{n-1}\right)
$$

a $\mathbb{Z}$-graded vector space. In view of (4.2), we have a bilinear multiplication "." on $A$ given by

$$
\begin{aligned}
& E_{m} / E_{m-1} \times E_{n} / E_{n-1} \rightarrow E_{m+n} / E_{m+n-1} \\
& \left(u+E_{m-1}\right) \cdot\left(v+E_{n-1}\right)=u_{-1} v+E_{m+n-1} .
\end{aligned}
$$

This makes $\operatorname{gr}_{E} V$ a non-associative algebra. It is clear that $\mathbf{1} \in E_{0}$ is the identity. Since $\mathcal{D}$ preserves $E_{m}$ for all $m$, we have a linear operator $\partial$ on $\operatorname{gr}_{E} V$ defined by

$$
\begin{aligned}
\partial: E_{m} / E_{m-1} & \rightarrow E_{m} / E_{m-1} \\
\left(u+E_{m-1}\right) & \mapsto \mathcal{D} u+E_{m-1} .
\end{aligned}
$$

Furthermore, in view of (4.3), for every $i \geq 0$, we have a well defined bilinear multiplication on $\operatorname{gr}_{E} V$ :

$$
\begin{aligned}
E_{m} / E_{m-1} \times E_{n} / E_{n-1} & \rightarrow E_{m+n-1} / E_{m+n-2} \\
\left(u+E_{m-1}, v+E_{n-1}\right) & \mapsto u_{i} v+E_{m+n-2} .
\end{aligned}
$$


Then for $u \in E_{m}, v \in E_{n}$ with $m, n \in \mathbb{Z}$ we define

$$
Y_{-}\left(u+E_{m-1}, x\right)\left(v+E_{n-1}\right)=\sum_{i \geq 0}\left(u_{i} v+E_{m+n-2}\right) x^{-i-1} \in x^{-1}\left(E_{m+n-1} / E_{m+n-2}\right)\left[x^{-1}\right] .(4 .
$$

We have:

Proposition 4.2 Let $(V, E)$ be a filtered vertex algebra and let $\operatorname{gr}_{E} V$ be the associated graded vector space defined in (4.5). Then $\operatorname{gr}_{E} V$ equipped with the structures $\left(\cdot, \partial, Y_{-}\right)$ defined in (4.6), (4.7) and (4.8) is a vertex Poisson algebra. Furthermore,

$$
\begin{aligned}
& \partial\left(\operatorname{gr}_{E} V\right)_{m} \subset\left(\operatorname{gr}_{E} V\right)_{m} \\
& a b \in\left(\operatorname{gr}_{E} V\right)_{m+n}=E_{m+n} / E_{m+n-1} \\
& a_{i} b \in\left(\operatorname{gr}_{E} V\right)_{m+n-1}=E_{m+n-1} / E_{m+n-2}
\end{aligned}
$$

for $m, n \in \mathbb{Z}, a \in E_{m} / E_{m-1}, b \in E_{n} / E_{n-1}, i \geq 0$.

Proof. First, we prove that $\left(\operatorname{gr}_{E} V, \cdot\right)$ is a commutative associative algebra with $\mathbf{1}+E_{-1}$ as the identity. Clearly, $\mathbf{1}+E_{0}$ is the identity for the algebra. For $u \in E_{m}, v \in E_{n}, m, n \in$ $\mathbb{Z}$, using (2.13), (4.2) and (4.3), we have

$$
u_{-1} v-v_{-1} u=u_{-1} v_{-1} \mathbf{1}-v_{-1} u_{-1} \mathbf{1}=\sum_{i \geq 0}\left(\begin{array}{c}
-1 \\
i
\end{array}\right)\left(u_{i} v\right)_{-2-i} \mathbf{1} \in E_{m+n-1} .
$$

This implies that $\operatorname{gr}_{E} V$ is commutative. Furthermore, let $u \in E_{m}, v \in E_{n}, w \in E_{k}$. Using the iterate formula (2.14) we have

$$
\left(u_{-1} v\right)_{-1} w=\sum_{i \geq 0}(-1)^{i}\left(\begin{array}{c}
-1 \\
i
\end{array}\right)\left(u_{-1-i} v_{-1+i} w-(-1)^{-1} v_{-2-i} u_{i} w\right) .
$$

Notice that from (4.3) and (4.2) we have $u_{-1-i} v_{-1+i} w \in E_{m+n+k-1}$ for $i \geq 1$ and $v_{-2-i} u_{i} w \in E_{m+n+k-1}$ for $i \geq 0$. Then from (4.14) we get

$$
\left(u_{-1} v\right)_{-1} w+E_{m+n+k-1}=u_{-1} v_{-1} w+E_{m+n+k-1} .
$$

This proves that $\operatorname{gr}_{E} V$ is associative, so that $\operatorname{gr}_{E} V$ is a commutative associative algebra.

We next prove that the operator $\partial$ is a derivation. Let $u \in E_{m}, v \in E_{n}$ with $m, n \in \mathbb{Z}$. Using (2.18) we get

$$
\begin{aligned}
& \partial\left(\left(u+E_{m-1}\right)\left(v+E_{n-1}\right)\right) \\
= & \mathcal{D}\left(u_{-1} v\right)+E_{m+n-1} \\
= & u_{-1} \mathcal{D} v+(\mathcal{D} u)_{-1} v+E_{m+n-1} \\
= & \left(u+E_{m-1}\right) \partial\left(v+E_{n-1}\right)+\left(\partial\left(u+E_{m-1}\right)\right)\left(v+E_{n-1}\right) .
\end{aligned}
$$

This proves that $\partial$ is a derivation of the commutative associative algebra $\operatorname{gr}_{E} V$. 
Now we check the axioms involving $Y_{-}$. Let $u \in E_{m}, v \in E_{n}, w \in E_{k}$. Then

$$
\begin{aligned}
& Y_{-}\left(u+E_{m-1}, x\right)\left(\left(v+E_{n-1}\right)\left(w+E_{k-1}\right)\right) \\
= & \sum_{i \geq 0}\left(u_{i} v_{-1} w+E_{m+n+k-2}\right) x^{-i-1} \\
= & \sum_{i \geq 0}\left(v_{-1} u_{i} w+E_{m+n+k-2}\right) x^{-i-1}+\sum_{i, j \geq 0}\left(\begin{array}{l}
i \\
j
\end{array}\right)\left(\left(u_{j} v\right)_{i-1-j} w+E_{m+n+k-2}\right) x^{-i-1} \\
= & \sum_{i \geq 0}\left(v_{-1} u_{i} w+\left(u_{i} v\right)_{-1} w+E_{m+n+k-2}\right) x^{-i-1} \\
& +\sum_{i \geq 0} \sum_{0 \leq j<i}\left(\begin{array}{l}
i \\
j
\end{array}\right)\left(\left(u_{j} v\right)_{i-1-j} w+E_{m+n+k-2}\right) x^{-i-1} \\
= & \sum_{i \geq 0}\left(v_{-1} u_{i} w+\left(u_{i} v\right)_{-1} w+E_{m+n+k-2}\right) x^{-i-1} \\
= & \left(v+E_{n-1}\right) Y_{-}\left(u+E_{m-1}, x\right)\left(w+E_{k-1}\right)+\left(Y_{-}\left(u+E_{m-1}, x\right)\left(v+E_{n-1}\right)\right)\left(w+E_{k-1}\right),
\end{aligned}
$$

since for $0 \leq j<i$,

$$
\left(u_{j} v\right)_{i-1-j} w \in E_{m+n+k-2} \text {. }
$$

This proves that $Y_{-}\left(u+E_{m-1}, x\right) \in x^{-1}\left(\operatorname{Der} \operatorname{gr}_{E} V\right)\left[\left[x^{-1}\right]\right]$. Thus

$$
Y_{-}(a, x) \in x^{-1}\left(\operatorname{Der}_{\operatorname{gr}_{E}} V\right)\left[\left[x^{-1}\right]\right] \quad \text { for } a \in \operatorname{gr}_{E} V \text {. }
$$

We also have

$$
\begin{aligned}
& \partial Y_{-}\left(u+E_{m-1}, x\right)\left(v+E_{n-1}\right) \\
= & \sum_{i \geq 0}\left(\mathcal{D} u_{i} v+E_{m+n-2}\right) x^{-i-1} \\
= & \sum_{i \geq 0}\left(u_{i} \mathcal{D} v+(\mathcal{D} u)_{i} v+E_{m+n-2}\right) x^{-i-1} \\
= & Y_{-}\left(u+E_{m-1}, x\right) \partial\left(v+E_{n-1}\right)+Y_{-}\left(\partial\left(u+E_{m-1}\right), x\right)\left(v+E_{n-1}\right) .
\end{aligned}
$$

Using the formula $\mathcal{D} u_{i} v=u_{i} \mathcal{D} v-i u_{i-1} v$ instead, we get

$$
\begin{aligned}
& \partial Y_{-}\left(u+E_{m-1}, x\right)\left(v+E_{n-1}\right) \\
= & \sum_{i \geq 0}\left(\mathcal{D} u_{i} v+E_{m+n-2}\right) x^{-i-1} \\
= & \sum_{i \geq 0}\left(u_{i} \mathcal{D} v-i u_{i-1} v+E_{m+n-2}\right) x^{-i-1} \\
= & Y_{-}\left(u+E_{m-1}, x\right) \partial\left(v+E_{n-1}\right)+\frac{d}{d x} Y_{-}\left(u+E_{m-1}, x\right)\left(v+E_{n-1}\right) .
\end{aligned}
$$

This proves that $\left[\partial, Y_{-}\left(u+E_{m-1}, x\right)\right]=Y_{-}\left(\partial\left(u+E_{m-1}\right), x\right)=\frac{d}{d x} Y_{-}\left(u+E_{m-1}, x\right)$. Thus

$$
\left[\partial, Y_{-}(a, x)\right]=Y_{-}(\partial a, x)=\frac{d}{d x} Y_{-}(a, x) \quad \text { for all } a \in \operatorname{gr}_{E} V
$$


Finally, for $u \in E_{m}, v \in E_{n-1}, w \in E_{k}$, using (2.13) we have

$$
\begin{aligned}
& {\left[Y_{-}\left(u+E_{m-1}, x_{1}\right), Y_{-}\left(v+E_{n-1}, x_{2}\right)\right]\left(w+E_{k-1}\right) } \\
= & \sum_{p, q \geq 0}\left(u_{p} v_{q} w-v_{q} u_{p} w+E_{m+n+k-3}\right) x_{1}^{-p-1} x_{2}^{-q-1} \\
= & \sum_{p, q \geq 0} \sum_{i=0}^{p}\left(\begin{array}{c}
p \\
i
\end{array}\right)\left(\left(u_{i} v\right)_{p+q-i} w+E_{m+n+k-3}\right) x_{1}^{-p-1} x_{2}^{-q-1} \\
= & \sum_{p, q \geq 0} \sum_{i=0}^{p}\left(\begin{array}{c}
p \\
i
\end{array}\right)\left(\left(u+E_{m-1}\right)_{i}\left(v+E_{n-1}\right)\right)_{p+q-i}\left(w+E_{k-1}\right) x_{1}^{-p-1} x_{2}^{-q-1},
\end{aligned}
$$

where $Y_{-}\left(u+E_{m-1}, x\right)=\sum_{p \geq 0}\left(u+E_{m-1}\right)_{p} x^{-p-1}$ with $\left(u+E_{m-1}\right)_{p} \in \operatorname{Der} \operatorname{gr}_{E} V$. This proves the half commutator formula for a vertex Lie algebra. Now the proof is complete.

A good filtration $E=\left\{E_{n}\right\}$ of a vertex algebra $V$ is said to be truncated if $E_{n}=0$ for $n$ sufficiently small. Typical truncated good filtrations are those with $E_{n}=0$ for $n<0$. (Note that for any good filtration $E$ of a nonzero vertex algebra $V, E_{0} \neq 0$, since $\mathbf{1} \in E_{0}$.)

Proposition 4.3 Let $V$ be a simple vertex algebra in the sense that there is no ideal other than $V$ and 0 . Then for any truncated good filtration of $V$ we have $E_{n}=0$ for $n<0$.

Proof. Suppose that $E=\left\{E_{n}\right\}$ is a good filtration of $V$ such that $E_{-k} \neq 0$ and $E_{n}=0$ for $n<-k$, where $k$ is a positive integer. For $u, v \in E_{-k}$, from (4.2) and (4.3) we have

$$
u_{m} v \in E_{-2 k}=0 \quad \text { for } m \in \mathbb{Z} \text {. }
$$

In particular,

$$
u_{m} u=0 \quad \text { for } u \in E_{-k}, m \in \mathbb{Z} .
$$

Thus each element $u$ of $E_{-k}$ is nilpotent in the sense of [LL]. By Proposition 3.10.6 of [LL], all nilpotent elements of $V$ form an ideal, which must equal $V$ because $E_{-k} \neq 0$ and $V$ is simple. This is a contradiction because $\mathbf{1}$ is not nilpotent.

Remark 4.4 Let $E=\left\{E_{n}\right\}$ be a good filtration of a vertex algebra $V$. For $u, v \in E_{0}$, from (4.2) and (4.3) we have

$$
\begin{aligned}
& u_{n} v \in E_{-1}\left(\subset E_{0}\right) \quad \text { for } n \geq 0, \\
& u_{n} v \in E_{0} \quad \text { for } n<0 .
\end{aligned}
$$

Consequently, $E_{0}$ is a vertex subalgebra of $V$ (notice that $\mathbf{1} \in E_{0}$ by assumption). Furthermore, $E_{-1}$ is an ideal of $E_{0}$, since for $u \in E_{0}, v \in E_{-1}$,

$$
u_{n} v, v_{n} u \in E_{-1} \quad \text { for } n \in \mathbb{Z} \text {. }
$$

It follows from (4.25) that $E_{0} / E_{-1}$ is a commutative vertex algebra. In particular, if $E_{-1}=0$, then $E_{0}$ is a commutative vertex subalgebra of $V$. 
We say a filtration $E=\left\{E_{n}\right\}$ for $V$ is finer than $E^{\prime}=\left\{E_{n}^{\prime}\right\}$, or $E^{\prime}$ is coarser than $E$, if $E_{n} \subset E_{n}^{\prime}$ for all $n \in \mathbb{Z}$.

Remark 4.5 For any vertex algebra $V$, we have a trivial filtration $E=\left\{E_{n}\right\}$ with $E_{n}=0$ for $n<0, E_{0}=\mathbb{C} 1$ and $E_{n}=V$ for $n \geq 1$. It is clear that this is a good filtration of $V$. Of course, it is the coarsest filtration. For this trivial filtration, we have $\operatorname{gr}_{E} V=E_{0} \oplus E_{1} / E_{0}=\mathbb{C} \mathbf{1} \oplus V / \mathbb{C} \mathbf{1}$, where $(V / \mathbb{C} \mathbf{1}) \cdot(V / \mathbb{C} \mathbf{1})=0$ and $\operatorname{gr}_{E} V=\mathbb{C} \mathbf{1} \oplus V / \mathbb{C} \mathbf{1}$ as a vertex Lie algebra is the direct sum of vertex Lie algebras $\mathbb{C}$ and $V / \mathbb{C} 1$, the quotient vertex Lie algebra of $V$ by the ideal $\mathbb{C} \mathbf{1}$ (cf. $[\mathrm{K}]$ ).

The following theorem gives a general construction and classification of good filtrations with $E_{n}=0$ for $n<0$ and $E_{0}=\mathbb{C} 1$ for a vertex algebra:

Theorem 4.6 Let $V$ be any vertex algebra. Let $U$ be a subspace of $V$ equipped with a vector space decomposition $U=\bigsqcup_{n \geq 1} U_{n}$. For $n \geq 0$, define $E_{n}^{U}$ to be the subspace of $V$ linearly spanned by the vectors

$$
u_{-n_{1}}^{(1)} \cdots u_{-n_{r}}^{(r)} \mathbf{1}
$$

for $r \geq 0, u^{(i)} \in U_{m_{i}}, n_{i} \geq 1$ with $m_{i} \geq 1$ and $m_{1}+\cdots+m_{r} \leq n$. Suppose that $U$ generates $V$ as a vertex algebra, i.e.,

$$
V=\operatorname{span}\left\{u_{n_{1}}^{(1)} \cdots u_{n_{r}}^{(r)} \mathbf{1} \mid \text { for } r \geq 0, u^{(i)} \in U, n_{i} \in \mathbb{Z}\right\},
$$

and suppose that

$$
u_{i} v \in E_{m+n-1}^{U} \quad \text { for } u \in U_{m}, v \in U_{n}, i \geq 0, m, n \geq 1
$$

i.e., for $u \in U_{m}, v \in U_{n}, i \geq 0, m, n \geq 1, u_{i} v$ is a linear combination of the vectors

$$
u_{-n_{1}}^{(1)} \cdots u_{-n_{r}}^{(r)} \mathbf{1}
$$

for $r \geq 0, u^{(i)} \in U_{m_{i}}, n_{i} \geq 1$ with $m_{1}+\cdots+m_{r} \leq m+n-1$. Then $E_{U}=\left\{E_{n}^{U}\right\}$ is a good filtration of $V$. In particular,

$$
V=\cup_{n \geq 0} E_{n}^{U}=\operatorname{span}\left\{u_{-n_{1}}^{(1)} \cdots u_{-n_{r}}^{(r)} \mathbf{1} \mid \text { for } r \geq 0, u^{(i)} \in U, n_{i} \geq 1\right\} .
$$

Furthermore, any good filtration $E=\left\{E_{n}\right\}$ of $V$ with $E_{n}=0$ for $n<0$ and with $E_{0}=\mathbb{C} \mathbf{1}$ can be obtained this way.

Proof. First, we prove that for any good filtration $E=\left\{E_{n}\right\}$ with the special property, there exists a subspace $U$ of $V$, equipped with a vector space decomposition $U=\coprod_{n \geq 1} U_{n}$, such that $E=E_{U}$ and such that (4.31) and (4.30) hold. For $n \geq 1$, let $U_{n}$ be a subspace of $E_{n}$ such that $E_{n}=U_{n} \oplus E_{n-1}$. Set $U=\coprod_{n>1} U_{n}$. (It is easy to see that the sum is direct.) We are going to prove that $E_{n}=E_{n}^{U}$ for all $n \geq 0$. Since $U_{m} \subset E_{m}$ for $m \geq 1$, it follows from the definition of $E_{n}^{U}$ and (4.3) that $E_{n}^{U} \subset E_{n}$ for $n \geq 1$. By 
definition, we also have $E_{0}=\mathbb{C} \mathbf{1}=E_{0}^{U}$. From definition, we have $U_{n} \subset E_{n}^{U}$ (since $u=u_{-1} 1$ ) and $E_{n}=U_{n}+E_{n-1}$ for $n \geq 1$. It follows from induction that $E_{n} \subset E_{n}^{U}$, so $E=E_{U}$. Since $\cup_{n \geq 0} E_{n}^{U}=\cup_{n \geq 0} E_{n}=V$, (4.31) holds. Furthermore, (4.30) holds because $u_{i} v \in E_{m+n-1}=E_{m+n-1}^{U}$.

Let $U$ be a subspace of $V$ equipped with a vector space decomposition $U=\prod_{n \geq 1} U_{n}$ such that (4.31) and (4.30) hold. From now on we shall just use $E_{n}$ for $E_{n}^{U}$. To prove that $E=\left\{E_{n}=E_{n}^{U}\right\}$ is a good filtration we must prove that $\cup_{n \geq 0} E_{n}=V$ and that for $n, p \geq 0, m \in \mathbb{Z}$ and for $v \in E_{p}$,

$$
\begin{array}{ll}
v_{m} E_{n} \subset E_{p+n-1} & \text { for } m \geq 0 \\
v_{m} E_{n} \subset E_{p+n} & \text { for } m<0 .
\end{array}
$$

Notice that (4.32) and (4.33) imply that $\cup_{n \geq 0} E_{n}$ is a vertex subalgebra of $V$. Since $U \subset \cup_{n \geq 0} E_{n}$ and $U$ generates $V$, (4.32) and (4.33) imply that $\cup_{n \geq 0} E_{n}=V$. Then it suffices to prove (4.32) and (4.33).

For $u \in U_{n}$ with $n \geq 1$, we say $u$ is homogeneous and we define $|u|=n$. First, we observe that from definition,

$$
u_{m} E_{n} \subset E_{n+|u|} \quad \text { for homogeneous } u \in U \text {, and for } m<0 \text {. }
$$

We also have

$$
\mathcal{D} E_{n} \subset E_{n} \quad \text { for } n \geq 0
$$

since $\left[\mathcal{D}, u_{-k}\right]=k u_{-k-1}$ and $\mathcal{D} \mathbf{1}=0$ for $u \in U, k \geq 1$.

We shall prove (4.32) and (4.33) by induction on $n+p$. If $p=0$ or $n=0$, it is clear because $E_{0}=\mathbb{C} \mathbf{1}$ and $v_{-k-1} \mathbf{1}=\frac{1}{k !} \mathcal{D}^{k} v \in E_{p}$ for $v \in E_{p}, k \geq 0$. Assume that $n, p \geq 1$. We only need to consider typical vectors

$$
v=v_{-k_{1}}^{(1)} \cdots v_{-k_{r}}^{(r)} \mathbf{1}
$$

for $r \geq 1, v^{(i)} \in U, k_{i} \geq 1$ with $\left|v^{(1)}\right|+\cdots+\left|v^{(r)}\right|=p$.

If $r \geq 2$, we have

$$
v=u_{-k} v^{\prime} \quad \text { where } u=v^{(1)} \in U, k=k_{1} \geq 1, v^{\prime} \in E_{p-|u|}
$$

with $|u|<p$. For $w \in E_{n}$, by the iterate formula we have

$$
v_{m} w=\sum_{i \geq 0}\left(\begin{array}{c}
-k \\
i
\end{array}\right)(-1)^{i}\left(u_{-k-i} v_{m+i}^{\prime} w-(-1)^{k+i} v_{-k+m-i}^{\prime} u_{i} w\right) .
$$

Noticing that $u \in E_{|u|}, v^{\prime} \in E_{p-|u|}$ and $|u|+n<p+n$, for $i \geq 0$, using the inductive hypothesis and (4.34) we have

$$
\begin{aligned}
& u_{-k-i} v_{m+i}^{\prime} w-(-1)^{k+i} v_{-k+m-i}^{\prime} u_{i} w \in E_{n+p-1} \quad \text { for } m \geq 0 \\
& u_{-k-i} v_{m+i}^{\prime} w-(-1)^{k+i} v_{-k+m-i}^{\prime} u_{i} w \in E_{n+p} \quad \text { for } m<0 .
\end{aligned}
$$


Then it follows that $v_{m} w \in E_{n+p-1}$ for $m \geq 0$ and $v_{m} w \in E_{n+p}$ for $m<0$.

Now, we consider the case that $r=1$. That is, $v=u_{-k} \mathbf{1}$ for some $u \in U, k \geq 1$ with $p=|u|$, so

$$
v_{m}=\left(u_{-k} \mathbf{1}\right)_{m}=\left(\begin{array}{c}
m \\
k-1
\end{array}\right) u_{m+1-k} \quad \text { for } m \in \mathbb{Z} .
$$

If $m<0$, then $m+1-k<0$, and if $0 \leq m \leq k-1$, then $v_{m}=0$ and if $m \geq k$, we have $m+1-k \geq 0$. In view of this, it suffices to consider $v=u$. Consider any vector $u_{-n_{1}}^{(1)} w \in E_{n}$ with $w \in E_{n-\left|u^{(1)}\right|}$. Then

$$
u_{m} u_{-n_{1}}^{(1)} w=u_{-n_{1}}^{(1)} u_{m} w+\sum_{i \geq 0}\left(\begin{array}{c}
m \\
i
\end{array}\right)\left(u_{i} u^{(1)}\right)_{m-n_{1}-i} w .
$$

Since $u \in E_{|u|}, w \in E_{n-\left|u^{(1)}\right|}$ and $|u|+n-\left|u^{(1)}\right|=p+n-\left|u^{(1)}\right|<n+p$, by inductive hypothesis, $u_{m} w \in E_{|u|+n-\left|u^{(1)}\right|}$ for $m<0$ and $u_{m} w \in E_{|u|+n-\left|u^{(1)}\right|-1}$ for $m \geq 0$, so that $u_{-n_{1}}^{(1)} u_{m} w \in E_{|u|+n}$ for $m<0$ and $u_{-n_{1}}^{(1)} u_{m} w \in E_{|u|+n-1}$ for $m \geq 0$. On the other hand, since, by assumption

$$
u_{i} u^{(1)} \in E_{|u|+\left|u^{(1)}\right|-1},
$$

by inductive hypothesis, we have $\left(u_{i} u^{(1)}\right)_{m-n_{1}-i} w \in E_{|u|+n-1}$ for all $i \geq 0, m \in \mathbb{Z}$. Thus $u_{m} u_{-n_{1}}^{(1)} w \in E_{n+p}$ for $m<0$ and $u_{m} u_{-n_{1}}^{(1)} w \in E_{n+p-1}$ for $m \geq 0$. This completes the inductive step and the proof.

For an illustration we next apply Theorem 4.6 to the vertex algebra associated with a vertex Lie algebra. Recall from Proposition 3.7 that for any vertex Lie algebra $R$, the symmetric algebra $S(R)$ has a vertex Poisson algebra structure. Furthermore, $S(R)$ is an $\mathcal{L}(R)_{+}$-module.

Proposition 4.7 Let $R$ be a vertex Lie algebra and let $\mathcal{V}(R)$ be the associated vertex algebra. Let $E=\left\{E_{n}\right\}$ be the sequence defined by

$$
E_{n}=\operatorname{span}\left\{a^{(1)}(-1) \cdots a^{(r)}(-1) \mathbf{1} \mid 0 \leq r \leq n, a^{(i)} \in R\right\} .
$$

Then $E=\left\{E_{n}\right\}$ is a good filtration of $\mathcal{V}(R)$ and each $E_{n}$ is an $\mathcal{L}(R)_{+}$-submodule of $\mathcal{V}(R)$. Furthermore, the linear map

$$
\begin{aligned}
\psi: S(R) & \rightarrow \operatorname{gr}_{E} \mathcal{V}(R) \\
u^{(1)} \cdots u^{(n)} & \mapsto u^{(1)}(-1) \cdots u^{(n)}(-1) \mathbf{1}+E_{n-1}
\end{aligned}
$$

for $u^{(i)} \in R$ is a vertex-Poisson-algebra isomorphism. Furthermore, $\psi$ is an $\mathcal{L}(R)_{+}$-module isomorphism.

Proof. Taking $U=U_{1}=R$ we have $E_{n}=E_{n}^{U}$ as defined in Theorem 4.6. Since $R$ generates $\mathcal{V}(R)$ as a vertex algebra and since $u_{i} v \in R$ for $u, v \in R, i \geq 0$, by Theorem $4.6 E$ is a good filtration. By (4.32) and (4.33) we have

$$
v_{m} E_{n} \subset E_{n} \quad \text { for } v \in R, m, n \geq 0 \text {. }
$$


Then each $E_{n}$ is an $\mathcal{L}(R)_{+}$-submodule of $\mathcal{V}(R)$.

It follows from Proposition 2.17 and the Poincaré-Birkhoff-Witt theorem that $\psi$ is a linear isomorphism (see [Di]) and then it follows from the definition of the multiplication of $\operatorname{gr}_{E} \mathcal{V}(R)$ that $\psi$ is an algebra isomorphism. Furthermore, for $u, v \in R$, we have

$$
\psi(\partial u)=\partial u+E_{0}=\mathcal{D} u+E_{0}=\partial\left(u+E_{0}\right)=\partial \psi(u),
$$

and

$$
\psi\left(Y_{-}(u, x) v\right)=\sum_{i \geq 0}\left(u_{i} v+E_{0}\right) x^{-i-1}=Y_{-}\left(u+E_{0}, x\right)\left(v+E_{0}\right)=Y_{-}(\psi(u), x) f(v) .
$$

By Lemma 3.3, $\psi$ is a vertex-Poisson-algebra homomorphism, so that it is a vertexPoisson-algebra isomorphism.

Note that the $\mathcal{L}(R)_{+}$-module structure on $S(R)$ is given by $u(x)^{-}=Y_{-}(u, x)$ for $u \in R$, where

$$
u(x)^{-}=\sum_{n \geq 0} u(n) x^{-n-1} \in \mathcal{L}(R)_{+}\left[\left[x^{-1}\right]\right]
$$

and $Y_{-}$is the vertex Lie algebra structure map of $S(R)$. On the other hand, the $\mathcal{L}(R)_{+^{-}}$ module structure on $\mathcal{V}(R)$ is given by $u(x)^{-}=Y(u, x)^{-}$for $u \in R$, where $Y$ is the vertex operator map of $\mathcal{V}(R)$ and $Y(u, x)^{-}=\operatorname{Sing} Y(u, x)$, so that the $\mathcal{L}(R)_{+}$-module structure on $\operatorname{gr}_{E} \mathcal{V}(R)$ is given by $u(x)^{-}=Y_{-}\left(u+E_{0}, x\right)$ for $u \in R=U \subset E_{1}$, where $Y_{-}$is the vertex Lie algebra structure map of $\operatorname{gr}_{E} \mathcal{V}(R)$. Now, with $\psi$ as a vertex-Poisson-algebra isomorphism, it follows immediately that $\psi$ is an $\mathcal{L}(R)_{+}$-module isomorphism.

Let $U$ be a subspace of a vertex algebra $V$. Following $[\mathrm{K}]$ we say that $U$ strongly generates $V$ if

$$
V=\operatorname{span}\left\{u_{-n_{1}}^{(1)} \cdots u_{-n_{r}}^{(r)} \mathbf{1} \mid r \geq 0, u^{(i)} \in U, n_{i} \geq 1\right\} .
$$

We say that $U$ generates $V$ with $P B W$ spanning property if for some basis $\left\{u^{(\alpha)} \mid \alpha \in I\right\}$ of $U$ and for any order ">" on the set $\left\{u_{-r}^{(\alpha)} \mid \alpha \in I, r \geq 1\right\}, V$ is linearly spanned by the vectors

$$
u_{-n_{1}}^{\left(\alpha_{1}\right)} \cdots u_{-n_{r}}^{\left(\alpha_{r}\right)} \mathbf{1}
$$

for $r \geq 0, \alpha_{i} \in I, n_{i} \geq 1$, with $u_{-n_{1}}^{\left(\alpha_{1}\right)} \geq \cdots \geq u_{-n_{r}}^{\left(\alpha_{r}\right)}$. In terms of this notion we have:

Theorem 4.8 Let $V$ be a vertex algebra and let $U$ be a generating subspace of $V$. Suppose that there exists a vector-space decomposition $U=\amalg_{n \geq 1} U_{n}$ such that for any $u \in U_{m}, v \in$ $U_{n}, i \geq 0, u_{i} v$ is a linear combination of the vectors

$$
u_{-n_{1}}^{(1)} \cdots u_{-n_{r}}^{(r)} 1
$$

for $r \geq 0, u^{(i)} \in U_{m_{i}}, n_{i} \geq 1$ with $m_{1}+\cdots+m_{r} \leq m+n-1$. Then $U$ generates $V$ with $P B W$ spanning property. 
Proof. By Theorem 4.6, we have a good filtration $E=\left\{E_{n}^{U}\right\}$ associated to $U$. In particular,

$$
V=\cup_{n \geq 0} E_{n}^{U}=\operatorname{span}\left\{u_{-n_{1}}^{(1)} \cdots u_{-n_{r}}^{(r)} \mathbf{1} \mid r \geq 0, u^{(i)} \in U, n_{i} \geq 1\right\} .
$$

Let $\left\{u^{(\alpha)} \mid \alpha \in I\right\}$ be a basis of $U$, consisting of homogeneous vectors. For $\alpha, \beta \in I, r, s \in$ $\mathbb{Z}$, from Borcherds' commutator formula we have

$$
\left[u_{r}^{(\alpha)}, u_{s}^{(\beta)}\right]=\sum_{i \geq 0}\left(\begin{array}{l}
r \\
i
\end{array}\right)\left(u_{i}^{(\alpha)} u^{(\beta)}\right)_{r+s-i}
$$

Furthermore, if $u^{(\alpha)} \in U_{m}\left(\subset E_{m}^{U}\right)$ and $u^{(\beta)} \in U_{n}\left(\subset E_{n}^{U}\right)$, by (4.3) we have

$$
u_{i}^{(\alpha)} u^{(\beta)} \in E_{m+n-1}^{U} \quad \text { for } i \geq 0 .
$$

With the property (4.2), it now follows immediately from the classical argument with the universal enveloping algebra of a Lie algebra (cf. [Di]), just as in [KL].

Remark 4.9 Notice that the vertex algebra $\mathcal{V}(R)$ associated with a vertex Lie algebra $R$ is generated by $U=R$ with linear relations while Theorems 4.6 and 4.8 concern vertex algebras generated by $U$ with general nonlinear generating relations.

We next apply Theorems 4.6 and 4.8 to "N-graded vertex algebras."

A $\mathbb{Z}$-graded vertex algebra is a vertex algebra equipped with a $\mathbb{Z}$-grading $V=\bigsqcup_{n \in \mathbb{Z}} V_{(n)}$ such that the following conditions hold for $u \in V_{(k)}, m, n, k \in \mathbb{Z}$ :

$$
u_{m} V_{(n)} \subset V_{(n+k-m-1)}
$$

An $\mathbb{N}$-graded vertex algebra is defined in the obvious way. For $v \in V_{(n)}$ for $n \in \mathbb{Z}$, we say $v$ is homogeneous of weight $n$ and we write wt $v=n$.

The following results were proved in [KL] (cf. [Li3]):

Theorem 4.10 Let $V=\coprod_{n \in \mathbb{N}} V_{(n)}$ be an $\mathbb{N}$-graded vertex algebra with $V_{(0)}=\mathbb{C} \mathbf{1}$. Denote by $C_{1}(V)$ the subspace of $V_{+}=\coprod_{n>0} V_{(n)}$ linearly spanned by the vectors $u_{-1} v$ for $u, v \in V_{+}$ and by the vectors $w_{-2} \mathbf{1}(=\mathcal{D} w)$ for $w \in V$. Then a graded subspace $U$ of $V_{+}$strongly generates $V$, i.e.,

$$
V=\operatorname{span}\left\{u_{-n_{1}}^{(1)} \cdots u_{-n_{r}}^{(r)} \mathbf{1} \mid r \geq 0, u^{(i)} \in U, n_{i} \geq 1\right\}
$$

if and only if $V_{+}=U+C_{1}(V)$. Furthermore, $U$ is a minimal graded strong generating subspace of $V$ if and only if $V_{+}=U \oplus C_{1}(V)$. If a graded subspace $U$ of $V_{+}$strongly generates $V$, then $U$ generates $V$ with $P B W$ spanning property.

Now we have: 
Theorem 4.11 Let $V=\bigsqcup_{n \in \mathbb{N}} V_{(n)}$ be an $\mathbb{N}$-graded vertex algebra with $V_{(0)}=\mathbb{C} \mathbf{1}$ and let $U$ be a graded subspace of $V_{+}=\bigsqcup_{n>0} V_{(n)}$. Then the following statements are equivalent:

(a) $U$ generates $V$ as a vertex algebra and for $u \in U_{m}=U \cap V_{(m)}, v \in U_{n}=$ $U \cap V_{(n)}, m, n \geq 1$ and for $i \geq 0, u_{i} v$ is a linear combination of the vectors

$$
u_{-n_{1}}^{(1)} \cdots u_{-n_{r}}^{(r)} 1
$$

for $r \geq 0, u^{(i)} \in U_{m_{i}}, n_{i} \geq 1$ with $m_{1}+\cdots+m_{r} \leq m+n-1$.

(b) $U$ strongly generates $V$, i.e.,

$$
V=\operatorname{span}\left\{u_{-k_{1}}^{(1)} \cdots u_{-k_{r}}^{(r)} \mathbf{1} \mid r \geq 0, u^{(i)} \in U, k_{i} \geq 1\right\} .
$$

(c) $V=U+C_{1}(V)$.

(d) $U$ generates $V$ with $P B W$ spanning property.

Furthermore, assume that any one of the four equivalent conditions holds and for $n \geq 0$, denote by $E_{n}^{U}$ the subspace of $V$ linearly spanned by the vectors

$$
u_{-k_{1}}^{(1)} \cdots u_{-k_{r}}^{(r)} \mathbf{1}
$$

for $r \geq 0, u^{(i)} \in U, k_{i} \geq 1$ with wt $u^{(1)}+\cdots+$ wt $u^{(r)} \leq n$. Then the sequence $E_{U}=\left\{E_{n}^{U}\right\}$ is a good filtration of vertex algebra $V$ and for $n \geq 0$,

$$
V_{(0)}+\cdots+V_{(n)} \subset E_{n}^{U} .
$$

Proof. From Theorem 4.10, (b) and (c) are equivalent, and (b) implies (d). Clearly, (d) implies (b). Thus (b), (c) and (d) are equivalent. In view of Theorem 4.6 (note that $\left.U=\coprod_{n \geq 1}\left(U \cap V_{(n)}\right)\right)$, (a) implies (b). Also in view of Theorem 4.6, it suffices to prove that (b) implies (a) and (4.49). First we prove that (b) implies (4.49). From our assumption, for $m \geq 0, V_{(m)}$ is linearly spanned by the vectors

$$
u_{-k_{1}}^{(1)} \cdots u_{-k_{r}}^{(r)} \mathbf{1}
$$

for homogeneous vectors $u^{(i)} \in U$ with

$$
m=\left(\operatorname{wt} u^{(1)}+k_{1}-1\right)+\cdots+\left(\text { wt } u^{(r)}+k_{r}-1\right) .
$$

Since $k_{i} \geq 1$, we have $m \geq$ wt $u^{(1)}+\cdots+$ wt $u^{(r)}$, so that by definition,

$$
u_{-k_{1}}^{(1)} \cdots u_{-k_{r}}^{(r)} 1 \in E_{m}^{U} .
$$

Thus $V_{(m)} \subset E_{m}^{U}$. We immediately have (4.49), since $E_{m}^{U} \subset E_{n}^{U}$ for $m \leq n$.

For any $u \in U_{(m)}, v \in U_{(n)}, m, n \geq 1, i \geq 0$, since wt $\left(u_{i} v\right)=$ wt $u+$ wt $v-i-1=$ $m+n-i-1$ and $V_{(m)} \subset E_{m}^{U}$ for all $m \geq 0$, we have

$$
u_{i} v \in V_{(m+n-i-1)} \subset E_{m+n-i-1}^{U} \subset E_{m+n-1}^{U} .
$$

This proves that (b) implies (a), completing the proof. 
Remark 4.12 Suppose that $E=\left\{E_{n}\right\}$ is a good filtration of $V$. We define $E^{\prime}=\left\{E_{n}^{\prime}\right\}$ by $E_{2 n}^{\prime}=E_{n}$ and $E_{2 n+1}^{\prime}=E_{n}$ for $n \geq 0$. Clearly, $E^{\prime}$ is an increasing filtration of $V$. It is straightforward to check that it is a good filtration. Thus, in general there does not exist a finest good filtration. Of course, good filtrations of a vertex algebra $V$ by no means are unique.

Despite of the non-uniqueness of good filtrations on a vertex algebra $V$ we are going to show that the good filtration $E^{U}=\left\{E_{n}^{U}\right\}$ associated with a graded subspace $U$ in fact does not depend on $U$. First, we have:

Lemma 4.13 Let $V=\coprod_{n \geq 0} V_{(n)}$ be an $\mathbb{N}$-graded vertex algebra with $V_{(0)}=\mathbb{C} \mathbf{1}$ and let $E=\left\{E_{n}\right\}$ be the filtration obtained in Theorem 4.11 from a graded subspace $U$ of $V_{+}$. Let $E^{\prime}=\left\{E_{n}^{\prime}\right\}$ be any good filtration for $V$ such that $V_{(n)} \subset E_{n}^{\prime}$ for $n \geq 0$. Then

$$
E_{n} \subset E_{n}^{\prime} \text { for all } n \geq 0 \text {. }
$$

Proof. By definition, $E_{0}=\mathbb{C} \mathbf{1} \subset E_{0}^{\prime}$. Assume $n \geq 1$. By definition, $E_{n}$ is linearly spanned by the vectors

$$
u_{-m_{1}}^{(1)} \cdots u_{-m_{r}}^{(r)} \mathbf{1}
$$

for $r \geq 1, u^{(i)} \in U, m_{i} \geq 1$ with wt $u^{(1)}+\cdots+$ wt $u^{(r)} \leq n$. For $1 \leq i \leq r$, by assumption

$$
u^{(i)} \in V_{\left(\mathrm{wt} u^{(i)}\right)} \subset E_{\mathrm{wt} u^{(i)}}^{\prime} .
$$

In view of $(4.2)$ we have

$$
u_{-n_{1}}^{(1)} \cdots u_{-n_{r}}^{(r)} \mathbf{1} \in E_{\mathrm{wt} u^{(1)}+\cdots+\mathrm{wt} u^{(r)}}^{\prime} \subset E_{n}^{\prime}
$$

Thus $E_{n} \subset E_{n}^{\prime} . \quad \square$.

Since for the filtration $E^{U}=\left\{E_{n}^{U}\right\}$ associated to any graded strong generating subspace $U, V_{(n)} \subset E_{n}$ for all $n \geq 0$ (by Theorem 4.11), it follows from Lemma 4.13 that the filtrations associated to any two graded generating subspaces must be the same. Therefore, we have proved:

Theorem 4.14 Let $V=\bigsqcup_{n \in \mathbb{N}} V_{(n)}$ be an $\mathbb{N}$-graded vertex algebra with $V_{(0)}=\mathbb{C} \mathbf{1}$. Then the filtration $E_{U}=\left\{E_{n}^{U}\right\}$ constructed in Theorem 4.11 from a graded strong generating subspace $U$ of $V$ does not depend on $U$. Furthermore, this filtration is the unique finest filtration with the property that $V_{(n)} \subset E_{n}$ for all $n \geq 0$.

We call the good filtration associated with the graded subspace $U=V_{+}$the standard filtration and we call the associated vertex Poisson algebra the standard vertex Poisson algebra associated with $V$, which we denote by gr $V$. In a sequel we shall use gr $V$ to study the vertex algebraic structure of $V$. 


\section{Formal deformation of vertex (Poisson) algebras}

In this section we first formulate a notion of $h$-adic vertex algebra and then using this notion we formulate a notion of formal deformation of vertex algebras and vertex Poisson algebras. We relate the construction of vertex Poisson algebras from filtered vertex algebras with Frenkel and Ben-Zvi's construction and we give a formal deformation of the vertex Poisson algebras $S(R)$ and $S_{\lambda}(R)$ associated with a vertex Lie algebra $R$.

In the literature, vertex algebras are often considered to be over $\mathbb{C}$ while most of the results naturally carry over for vertex algebras over a field of characteristic 0, or over a unital commutative associative algebra over a field of characteristic 0 . In this section we shall concern vertex algebras over $\mathbb{C}$, or over a unital commutative associative algebra $K$ over $\mathbb{C}$, e.g., $K=\mathbb{C}[h]$, the polynomial algebra for a formal variable $h$.

Let $V$ be a vertex algebra over $\mathbb{C}$. Then $V[h]=V \otimes \mathbb{C}[h]$ is naturally a vertex algebra over $\mathbb{C}[h]$ and $V \otimes \mathbb{C}[[h]]$ is naturally a vertex algebra over $\mathbb{C}[[h]]$. Consider the space $V[[h]]$ of all formal power series in $h$. (Notice that $V \otimes \mathbb{C}[[h]]$ is a proper subspace of $V[[h]]$ unless $V$ is finite-dimensional.) It is well known that $V[[h]]$ is the completion of the $\mathbb{C}[h]$-module $V[h]$ with respect to the $h$-adic topology. Formally extend the vertex operator map $Y$ of vertex algebra $V$ to a $\mathbb{C}[[h]]$-linear map from $V[[h]]$ to $($ End $V[[h]])\left[\left[x, x^{-1}\right]\right]$ by

$$
Y(a(h), x) b(h)=\sum_{m, n \geq 0} Y(a(m), x) b(n) h^{m+n}\left(\in V[[h]]\left[\left[x, x^{-1}\right]\right]\right)
$$

for $a(h)=\sum_{n \geq 0} a(n) h^{n}, b(h)=\sum_{n \geq 0} b(n) h^{n} \in V[[h]]$. Because $Y(a(h), x) b(h)$ in general involves infinitely many negative powers of $x$, the triple $(V[[h]], Y, \mathbf{1})$ does not carry the structure of a vertex algebra (over $\mathbb{C}[h]$ or $\mathbb{C}[[h]]$ ) in the precise sense. On the other hand, note that for any nonnegative integer $n$, the quotient space $V[[h]] / h^{n} V[[h]]$ is naturally a vertex algebra (over $\mathbb{C}[[h]]$, or $\mathbb{C}[h]$ ), which is isomorphic to the quotient vertex algebra $V[h] / h^{n} V[h]$ over $\mathbb{C}[h]$. Motivated by this we formulate the following notion of $h$-adic vertex algebra:

Definition 5.1 An $h$-adic vertex algebra is a $\mathbb{C}[[h]]$-module $A[[h]]$, where $A$ is a vector space over $\mathbb{C}$, equipped with the $h$-adic topology, a distinguished vector $\mathbf{1} \in A[[h]]$ and a continuous $\mathbb{C}[[h]]$-linear map $Y_{h}$ from $A[[h]] \otimes A[[h]]$ to $A[[h]]\left[\left[x, x^{-1}\right]\right]$ such that for every nonnegative integer $n$, the triple $\left(A[[h]] / h^{n} A[[h]], \bar{Y}_{(n)}, \mathbf{1}+h^{n} A[[h]]\right)$ carries the structure of a vertex algebra over $\mathbb{C}[[h]]$, where $\bar{Y}_{(n)}$ is the natural quotient map of $Y_{h}$, i.e.,

$$
\bar{Y}_{(n)}\left(a+h^{n} A[[h]], x\right)\left(b+h^{n} A[[h]]\right)=\sum_{m \in \mathbb{Z}}\left(a_{m} b+h^{n} A[[h]]\right) x^{-m-1}
$$

for $a, b \in A$, where $Y_{h}(a, x) b=\sum_{m \in \mathbb{Z}} a_{m} b x^{-m-1}$.

Remark 5.2 Let $A[[h]]$ be an $h$-adic vertex algebra. For every pair of nonnegative integers $i$ and $j$ with $i \leq j$, it is clear that the natural map $\theta_{i j}$ from $A[[h]] / h^{j} A[[h]]$ onto $A[[h]] / h^{i} A[[h]]$ is a vertex algebra homomorphism over $\mathbb{C}[[h]]$. The vertex algebras $A[[h]] / h^{n} A[[h]]$ (over $\mathbb{C}[[h]]$ ) together with these vertex algebra morphisms form an inverse system of vertex algebras over $\mathbb{C}[[h]]$. Then the $h$-adic vertex algebra $A[[h]]$ can be thought of as an inverse limit of the vertex algebras $A[[h]] / h^{n} A[[h]]$ over $\mathbb{C}[[h]]$. 
Proposition 5.3 Let $A$ be a vector space over $\mathbb{C}$. An h-adic vertex algebra structure on $A[[h]]$ amounts to a distinguished vector $\mathbf{1} \in A[[h]]$ and a continuous $\mathbb{C}[[h]]$-linear map $Y_{h}$ from $A[[h]] \otimes A[[h]]$ to $A[[h]]\left[\left[x, x^{-1}\right]\right]$ such that the following conditions hold: For $a \in A[[h]]$,

$$
\begin{aligned}
& Y_{h}(\mathbf{1}, x) a=a, \\
& Y_{h}(a, x) \mathbf{1} \in A[[h]][[x]] \quad \text { and } \quad \lim _{x \rightarrow 0} Y_{h}(a, x) \mathbf{1}=a ;
\end{aligned}
$$

for $a, b \in A[[h]], n \in \mathbb{N}$, there exists an integer $k$ such that

$$
a_{m} b \in h^{n} A[[h]] \quad \text { for } m \geq k
$$

and ( $h$-adic weak commutativity) for any $a, b, c \in A[[h]]$ and for any nonnegative integer $n$, there exists a nonnegative integer $k$ (depending on $a, b$ and $n$ ) such that

$$
\left(x_{1}-x_{2}\right)^{k}\left[Y_{h}\left(a, x_{1}\right), Y_{h}\left(b, x_{2}\right)\right] c \in h^{n} A[[h]]\left[\left[x_{1}, x_{1}^{-1}, x_{2}, x_{2}^{-1}\right]\right]
$$

and ( $h$-adic weak associativity) for any $a, b, c \in A[[h]]$ and for any nonnegative integer $n$, there exists a nonnegative integer $l$ (depending on $a, c$ and $n$ ) such that

$$
\left(x_{0}+x_{2}\right)^{l}\left(Y_{h}\left(a, x_{0}+x_{2}\right) Y_{h}\left(b, x_{2}\right) c-Y_{h}\left(Y_{h}\left(a, x_{0}\right) b, x_{2}\right) c\right) \in h^{n} A[[h]]\left[\left[x_{0}, x_{0}^{-1}, x_{2}, x_{2}^{-1}\right]\right] .
$$

Proof. Notice that we have

$$
\cap_{n \geq 0} h^{n} A[[h]]=0 .
$$

In view of this, for $a \in A[[h]], Y_{h}(\mathbf{1}, x) a=a$ if and only if for every positive integer $n, Y_{h}(a, x) a-a \in h^{n} A[[h]]\left[\left[x, x^{-1}\right]\right]$, which is equivalent to $\bar{Y}_{n}\left(\mathbf{1}+h^{n} A[[h]], x\right)(a+$ $\left.h^{n} A[[h]]\right)=a+h^{n} A[[h]]$. Similarly, the equivalence on the creation property is clear. As the Jacobi identity for a vertex algebra (over $\mathbb{C}[[h]]$ ) amounts to weak commutativity and weak associativity (see [DL], [Li2], [LL]), the Jacobi identity for vertex algebras $\left(A[[h]] / h^{n} A[[h]], \bar{Y}_{n}, \mathbf{1}+h^{n} A[[h]]\right)$ for $n \geq 1$ amounts to the $h$-adic weak commutativity and associativity.

Remark 5.4 Notice that in view of (5.5) the following Jacobi identity for $Y_{h}$

$$
\begin{aligned}
& x_{0}^{-1} \delta\left(\frac{x_{1}-x_{2}}{x_{0}}\right) Y_{h}\left(a, x_{1}\right) Y_{h}\left(b, x_{2}\right) c-x_{0}^{-1} \delta\left(\frac{x_{2}-x_{1}}{-x_{0}}\right) Y_{h}\left(b, x_{2}\right) Y_{h}\left(a, x_{1}\right) c \\
= & x_{2}^{-1} \delta\left(\frac{x_{1}-x_{0}}{x_{2}}\right) Y_{h}\left(Y_{h}\left(a, x_{0}\right) b, x_{2}\right) c
\end{aligned}
$$

holds in $A[[h]]\left[\left[x_{0}^{ \pm 1}, x_{1}^{ \pm 1}, x_{2}^{ \pm 1}\right]\right]$, where for $m, n, k \in \mathbb{Z}$, the coefficients of $x_{0}^{m} x_{1}^{n} x_{2}^{k}$ in the three main terms are infinite convergent sums in $A[[h]]$, unlike the coefficients of $x_{0}^{m} x_{1}^{n} x_{2}^{k}$ in the usual (algebraic) Jacobi identity, which are finite sums. 
Remark 5.5 In [EK], Etingof and Kazhdan introduced a notion of braided VOA over $\mathbb{C}[[h]]$. In fact, one can show that $h$-adic vertex algebras are exactly those braided VOA's over $\mathbb{C}[[h]]$ with $\mathcal{S}=1$.

Let $U$ be a vertex algebra over $\mathbb{C}[h]$, or an $h$-adic vertex algebra over $\mathbb{C}[[h]]$. Then $h U$ is an ideal of $U$, so that $U / h U$ is a vertex algebra over $\mathbb{C}[h]$ (with $h$ acting as zero). We may naturally consider $U / h U$ as a vertex algebra over $\mathbb{C}$. The following result is due to $[\mathrm{FB}]$ :

Proposition 5.6 Let $U$ be either an $h$-adic vertex algebra over $\mathbb{C}[[h]]$ or a vertex algebra over $\mathbb{C}[h]$ such that $\operatorname{ker}_{U} h \subset h U$. Assume that that $U / h U$ is a commutative vertex algebra. Then $\left(U / h U, \cdot, Y_{-}, \partial\right)$ carries the structure of a vertex Poisson algebra where

$$
\begin{aligned}
& (u+h U) \cdot(v+h U)=u_{-1} v+h U \\
& Y_{-}(u+h U, x)(v+h U)=\frac{1}{h} \operatorname{Sing} Y(u, x) v+h U\left[\left[x, x^{-1}\right]\right] \\
& \partial(u+h U)=\mathcal{D} u+h U=u_{-2} \mathbf{1}+h U
\end{aligned}
$$

for $u, v \in U$.

Remark 5.7 Proposition 5.6 is a variant of Proposition 15.2.4 of [FB] which states that if $V^{\epsilon}$ is a vertex algebra over $\mathbb{C}[\epsilon] /\left(\epsilon^{2}\right)$ and a flat module over $\mathbb{C}[\epsilon] /\left(\epsilon^{2}\right)$ such that $V^{0}=V^{\epsilon} / \epsilon V^{\epsilon}$ is commutative, then $V^{0}$ naturally acquires the structure of a vertex Poisson algebra. The variant Proposition 5.6 follows from the same proof of $[\mathrm{FB}]$ where the assumption ker $h \subset h U$ guarantees that $Y_{-}(u+h U, x)(v+h U)=\frac{1}{h} \operatorname{Sing} Y(u, x) v+h U\left[\left[x, x^{-1}\right]\right]$ is well defined.

Proposition 5.6 gives another way to construct vertex Poisson algebras (over $\mathbb{C}$ ) from vertex algebras (over $\mathbb{C}[[h]]$ ) (recall Proposition 4.2). In the next proposition we show that the vertex Poisson algebra $\operatorname{gr}_{E} V$ associated to a filtered vertex algebra $(V, E)$ can be realized as a vertex Poisson algebra $U / h U$ for some vertex algebra $U$ over $\mathbb{C}[h]$.

The following result is classical in nature:

Proposition 5.8 Let $(V, E)$ be a filtered vertex algebra over $\mathbb{C}$. For $n \geq 0$, let $U_{n}$ be a subspace of $E_{n}$ such that $E_{n}=U_{n} \oplus E_{n-1}$, so that

$$
V=U_{0} \oplus U_{1} \oplus \cdots=\coprod_{n \geq 0} U_{n} .
$$

For $n \geq 0$, denote by $p_{n}$ the projection map of $V$ onto $U_{n}$. Set

$$
V_{E}[h]=V[h]=\mathbb{C}[h] \otimes V,
$$

as a $\mathbb{C}[h]$-module, which is free. For $u \in U_{m}, v \in U_{n}$, we define

$$
Y_{h}(u, x) v=\sum_{j=0}^{m+n} h^{m+n-j} p_{j}(Y(u, x) v)=\sum_{r \in \mathbb{Z}} \sum_{j=0}^{m+n} h^{m+n-j} p_{j}\left(u_{r} v\right) x^{-r-1},
$$


and then extend the definition $\mathbb{C}[h]$-linearly to $V_{E}[h]$. Then $\left(V_{E}[h], Y_{h}, \mathbf{1}\right)$ carries the structure of a vertex algebra over $\mathbb{C}[h]$ such that $V_{E}[h] / h V_{E}[h]$ is commutative. Furthermore, the linear map

$$
\begin{aligned}
\psi: V & \rightarrow V_{E}[h] /(h-1) V_{E}[h] \\
v & \mapsto v+(h-1) V_{E}[h]
\end{aligned}
$$

is a vertex algebra isomorphism over $\mathbb{C}$ and the linear map

$$
\begin{aligned}
\phi: \operatorname{gr}_{E} V=\coprod_{m \geq 0} E_{m} / E_{m-1} & \rightarrow V_{E}[h] / h V_{E}[h] \\
v+E_{m-1} \in E_{m} / E_{m-1} & \mapsto p_{m}(v)+h V_{E}[h]
\end{aligned}
$$

is a vertex Poisson algebra isomorphism over $\mathbb{C}$.

Proof. First, for every $n \geq 0$, we have

$$
E_{n}=U_{0} \oplus \cdots \oplus U_{n}
$$

so that

$$
v=\sum_{k \geq 0} p_{k}(v)=\sum_{k=0}^{n} p_{k}(v) \quad \text { for } v \in E_{n} .
$$

Second, in view of properties (4.2) and (4.3) we have

$$
Y(u, x) v \in E_{m+n}((x)) \quad \text { for } u \in E_{m}, v \in E_{n} .
$$

Third, $\mathbb{C}[h] \otimes V$ is naturally a vertex algebra over $\mathbb{C}[h]$.

Let $\theta$ be the $\mathbb{C}[h]$-linear endomorphism of $V[h]=\mathbb{C}[h] \otimes V$ defined by

$$
\theta(f(h) v)=\sum_{n \geq 0} f(h) h^{n} p_{n}(v) \quad \text { for } f(h) \in \mathbb{C}[h], v \in V .
$$

In particular,

$$
\theta(u)=h^{n} u \quad \text { for } u \in U_{n}, n \geq 0 .
$$

It is easy to see that $\theta$ is injective. The image of $\theta$, which is $\sum_{n \geq 0} \mathbb{C}[h] h^{n} U_{n}$, is a vertex subalgebra (over $\mathbb{C}[h]$ ) because for $u \in U_{m}, v \in U_{n}$,

$$
\begin{gathered}
Y\left(h^{m} u, x\right)\left(h^{n} v\right)=h^{m+n} Y(u, x) v=\sum_{k=0}^{m+n} h^{m+n} p_{k}(Y(u, x) v) \\
=\sum_{k=0}^{m+n} h^{m+n-k} h^{k} p_{k}(Y(u, x) v) .
\end{gathered}
$$


Define a $\mathbb{C}[h]$-linear map $Y_{h}^{\prime}$ from $V[h]$ to $\left(\operatorname{End}_{\mathbb{C}[h]} V[h]\right)\left[\left[x, x^{-1}\right]\right]$ by

$$
Y_{h}^{\prime}(u, x) v=\theta^{-1} Y(\theta(u), x) \theta(v) \quad \text { for } u, v \in V[h] .
$$

Then $\left(V[h], Y_{h}^{\prime}, \mathbf{1}\right)$ carries the structure of a vertex algebra over $\mathbb{C}[h]$, which is transported from the vertex algebra $\theta(V[h])$ through the map $\theta$. Furthermore, for $u \in U_{m}, v \in U_{n}$, we have

$$
\begin{aligned}
Y_{h}^{\prime}(u, x) v & =\theta^{-1} Y(\theta(u), x) \theta(v)=\theta^{-1}\left(Y\left(h^{m} u, x\right)\left(h^{n} v\right)\right) \\
& =\sum_{k=0}^{m+n} \theta^{-1}\left(h^{m+n} p_{k}(Y(u, x) v)\right) \\
& =\sum_{k=0}^{m+n} h^{m+n-k} p_{k}(Y(u, x) v) \\
& =Y_{h}(u, x) v .
\end{aligned}
$$

This shows that $Y_{h}=Y_{h}^{\prime}$. We also have $\theta(\mathbf{1})=\mathbf{1}$ because $\mathbf{1} \in E_{0}=U_{0}$. Therefore, $\left(V_{E}[h], Y_{h}, \mathbf{1}\right)$ carries the structure of a vertex algebra over $\mathbb{C}[h]$. With $(h-1) V_{E}[h]$ being an ideal of $V_{E}[h], V_{E}[h] /(h-1) V_{E}[h]$ is naturally a vertex algebra over $\mathbb{C}[h]$. Clearly, the linear map $\psi$ from $V$ to $V_{E}[h] /(h-1) V_{E}[h]$ is a linear isomorphism sending 1 to $\mathbf{1}+(h-1) V_{E}[h]$. Furthermore, for $\in U_{m}, v \in U_{n}$, we have

$$
Y(u, x) v=\sum_{k=0}^{m+n} p_{k}(Y(u, x) v)=\left.Y_{h}(u, x) v\right|_{h=1}
$$

Thus $\psi$ is a vertex algebra isomorphism.

For $\in U_{m}, v \in U_{n}$, since $u_{r} v \in E_{m+n-1}$ for $r \geq 0$, we have $p_{m+n}\left(u_{r} v\right)=0$ for $r \geq 0$, so that

$$
Y_{h}(u, x) v=\sum_{r<0} \sum_{k=0}^{m+n} h^{m+n-k} p_{k}\left(u_{r} v\right) x^{-r-1}+h \sum_{r \geq 0} \sum_{k=0}^{m+n-1} h^{m+n-k-1} p_{k}\left(u_{r} v\right) x^{-r-1} .
$$

Thus $Y_{h}(u, x) v \in V_{h}[[x]]+h V_{h}((x))$. It follows that the quotient vertex algebra $V_{E}[h] / h V_{E}[h]$ is commutative. Recall that $E_{m}=U_{m} \oplus E_{m-1}$ for $m \geq 0$. For $u \in U_{m}=E_{m} / E_{m-1}, v \in$ $U_{n}=E_{n} / E_{n-1}$, we have

$$
\begin{aligned}
Y_{-}\left(u+E_{m-1}, x\right)\left(v+E_{n-1}\right) & =\sum_{r \geq 0}\left(u_{r} v+E_{m+n-2}\right) x^{-r-1} \\
& =\sum_{r \geq 0}\left(p_{m+n-1}\left(u_{r} v\right)+E_{m+n-2}\right) x^{-r-1}
\end{aligned}
$$

and

$$
\begin{aligned}
Y_{-}\left(u+h V_{E}[h], x\right)\left(v+h V_{E}[h]\right) & =\frac{1}{h} \operatorname{Sing} Y_{h}(u, x) v+h V_{E}[h] \\
& =\sum_{r \geq 0} \sum_{k=0}^{m+n-1} h^{m+n-k-1}\left(p_{k}\left(u_{r} v\right)+h V_{E}[h]\right) x^{-r-1} \\
& =\sum_{r \geq 0}\left(p_{m+n-1}\left(u_{r} v\right)+h V_{E}[h]\right) x^{-r-1} .
\end{aligned}
$$


We also have

$$
\left(u+E_{m-1}\right) \cdot\left(v+E_{n-1}\right)=u_{-1} v+E_{m+n-1}=p_{m+n}\left(u_{-1} v\right)+E_{m+n-1}
$$

and

$$
\begin{aligned}
& \left(u+h V_{E}[h]\right) \cdot\left(v+V_{E}[h]\right)\left(=\operatorname{Res}_{x} x^{-1} Y_{h}(u, x) v+h V_{E}[h]\right) \\
= & \sum_{i=0}^{m+n} h^{m+n-k} p_{k}\left(u_{-1} v\right)+h V_{E}[h] \\
= & p_{m+n}\left(u_{-1} v\right)+h V_{E}[h] .
\end{aligned}
$$

Now it is clear that $\phi$ is a vertex Poisson algebra isomorphism (note that $1 \in U_{0}=E_{0}$ ).

Remark 5.9 Technically speaking, $V_{E}[h]$ is not a well defined function of $(V, E)$ because the vertex algebra $V_{E}[h]$ also depends on the decompositions $E_{n}=\coprod_{i=0}^{n} U_{n}$. However, it is straightforward to check that different decompositions give rise to isomorphic vertex algebras $V_{E}[h]$.

Remark 5.10 Let $(V, E)$ be a filtered vertex algebra. Consider the subspace

$$
U=\coprod_{n \in \mathbb{N}} E_{n} h^{n} \subset V[h] .
$$

Since $E_{n} \subset E_{n+k}$ for $n, k \geq 0$, we have

$$
E_{n} h^{n+k} \subset E_{n+k} h^{n+k} \subset U
$$

so that $U$ is indeed a $\mathbb{C}[h]$-subspace of $V[h]$. In view of (5.19), $U$ is a vertex subalgebra of $V[h]$. Clearly, ker $h=0 \subset h U$. For $u \in E_{m}, v \in E_{n}, m, n, i \geq 0$, we have

$$
h^{m+n} u_{i} v \in h^{m+n} E_{m+n-1}=h\left(h^{m+n-1} E_{m+n-1}\right),
$$

Thus $U / h U$ is commutative. We have

$$
U / h U=\coprod_{n \geq 0} E_{n} h^{n} / E_{n-1} h^{n}=\coprod_{n \geq 0} E_{n} / E_{n-1}=\operatorname{gr}_{E} V
$$

as a vector space over $\mathbb{C}$. (This is exactly the classical construction (see $[\mathrm{CG}]$ ).) Similar to what we did in the proof of Proposition 5.8, one can show that $U / h U=\operatorname{gr}_{E} V$ as a vertex Poisson algebra and one can also show that the evaluation map with $h=1$ from $V[h]$ to $V$ gives rise to a vertex algebra isomorphism from $U /(h-1) U$ onto $V$. But, to identify $U$ with $V[h]$ one will have to use uncanonical decompositions $E_{n}=E_{n-1} \oplus U_{n}$ for $n \geq 0$ just as in Proposition 5.8. 
Let $\left(R, Y_{-}, \partial\right)$ be a vertex Lie algebra over $\mathbb{C}$. Extend $\partial$ to a $\mathbb{C}[h]$-linear endomorphism of $R[h]$ and extend $Y_{-}$to a $\mathbb{C}[h]$-bilinear map on $R[h] \otimes R[h]$. Then $\left(R[h], Y_{-}, \partial\right)$ is a vertex Lie algebra over $\mathbb{C}[h]$. It is straightforward to see that $\left(R[h], h Y_{-}, \partial\right)$ is a vertex Lie algebra over $\mathbb{C}[h]$, just as with Lie algebras. Let $\mathcal{V}_{h}(R)$ be the corresponding vertex algebra over $\mathbb{C}[h]$ associated to the vertex Lie algebra $\left(R[h], h Y_{-}, \partial\right)$ and let $Y_{h}$ denote the vertex operator map:

$$
Y_{h}: \mathcal{V}_{h}(R) \rightarrow \operatorname{Hom}_{\mathbb{C}[h]}\left(\mathcal{V}_{h}(R), \mathcal{V}_{h}(R)((x))\right)
$$

As with $\mathcal{V}(R), R$ is a $\mathbb{C}$-subspace of $\mathcal{V}_{h}(R)$. So we have natural $\mathbb{C}$-linear maps from $R$ to any quotient spaces of $\mathcal{V}_{h}(R)$. Now we have:

Theorem 5.11 For any vertex Lie algebra $\left(R, Y_{-}, \partial\right)$ (over $\left.\mathbb{C}\right)$, there is a unique vertex algebra isomorphism from $\mathcal{V}(R)$ onto the quotient vertex algebra $\mathcal{V}_{h}(R) /(h-1) \mathcal{V}_{h}(R)$, extending the natural map from $R$ to $\mathcal{V}_{h}(R) /(h-1) \mathcal{V}_{h}(R)$. The quotient vertex algebra $\mathcal{V}_{h}(R) / h \mathcal{V}_{h}(R)$ is commutative and there is a unique vertex Poisson algebra isomorphism from $S(R)$ onto the vertex Poisson algebra $\mathcal{V}_{h}(R) / h \mathcal{V}_{h}(R)$ obtained in Proposition 5.6, extending the natural map from $R$ to $\mathcal{V}_{h}(R) / h \mathcal{V}_{h}(R)$.

Proof. For $a, b \in R \subset R[h]$, we have

$$
\begin{aligned}
& \operatorname{Sing} Y_{h}(a, x) b=h Y_{-}(a, x) b=Y_{-}(a, x) b+(h-1) Y_{-}(a, x) b \\
& \partial a=\mathcal{D} a,
\end{aligned}
$$

where $\mathcal{D}$ denotes the $\mathcal{D}$-operator of the vertex algebra $\mathcal{V}_{h}(R)$. Thus, the $\mathbb{C}$-linear map $f: a \mapsto a+(h-1) \mathcal{V}_{h}(R)$ from $R$ to $\mathcal{V}_{h}(R) /(h-1) \mathcal{V}_{h}(R)$ is a vertex Lie algebra homomorphism. In view of Primc's result (Theorem 2.16), $f$ uniquely extends to a vertex algebra homomorphism $\bar{f}$ from $\mathcal{V}(R)$ to $\mathcal{V}_{h}(R) /(h-1) \mathcal{V}_{h}(R)$. It follows from the PoincaréBirkhoff-Witt theorem that $\bar{f}$ is an isomorphism.

For $a, b \in R \subset R[h]$, we have

$$
\begin{aligned}
{\left[Y_{h}\left(a, x_{1}\right), Y_{h}\left(b, x_{2}\right)\right] } & =\operatorname{Res}_{x_{0}} x_{2}^{-1} \delta\left(\frac{x_{1}-x_{0}}{x_{2}}\right) Y_{h}\left(Y_{h}\left(a, x_{0}\right) b, x_{2}\right) \\
& =\operatorname{Res}_{x_{0}} x_{2}^{-1} \delta\left(\frac{x_{1}-x_{0}}{x_{2}}\right) Y_{h}\left(\operatorname{Sing}_{x_{0}} Y_{h}\left(a, x_{0}\right) b, x_{2}\right) \\
& =\operatorname{Res}_{x_{0}} x_{2}^{-1} \delta\left(\frac{x_{1}-x_{0}}{x_{2}}\right) h Y_{h}\left(Y_{-}\left(a, x_{0}\right) b, x_{2}\right) .
\end{aligned}
$$

Since $R$ is a generating space of $\mathcal{V}_{h}(R),\left\{a+h \mathcal{V}_{h}(R) \mid a \in R\right\}$ is a generating subspace of $\mathcal{V}_{h}(R) / h \mathcal{V}_{h}(R)$. Notice that if $S$ is a generating subset of a vertex algebra $V$ such that $\left[Y\left(u, x_{1}\right), Y\left(v, x_{2}\right)\right]=0$ for $u, v \in S$, then $V$ is commutative. In view of this and (5.37), $\mathcal{V}_{h}(R) / h \mathcal{V}_{h}(R)$ is commutative. In view of Proposition 5.6, $\mathcal{V}_{h}(R) / h \mathcal{V}_{h}(R)$ is naturally a vertex Poisson algebra. Since

$$
\frac{1}{h} \operatorname{Sing} Y_{h}(a, x) b=Y_{-}(a, x) b \quad \text { for } a, b \in R,
$$


the map $g: a \mapsto a+h \mathcal{V}_{h}(R)$ from $R$ to $\mathcal{V}_{h}(R) / h \mathcal{V}_{h}(R)$ is a vertex Lie algebra homomorphism. It follows from Proposition 3.9 that $g$ uniquely extends to a vertex Poisson algebra homomorphism $\bar{g}$ from $S(R)$ into $\mathcal{V}_{h}(R) / h \mathcal{V}_{h}(R)$. Again, it follows from the Poincaré-Birkhoff-Witt theorem that $\bar{g}$ is an isomorphism.

Using the same arguments (with Remark 2.23 in place of Theorem 2.16) we have the following generalization:

Theorem 5.12 Let $\left(R, Y_{-}, \partial\right)$ be a vertex Lie algebra and let $\lambda$ be a partially defined linear functional on $\operatorname{ker}_{R} \partial$. Define $\mathcal{V}_{h}^{\lambda}(R)$ to be the quotient vertex algebra of $\mathcal{V}_{h}(R)$ modulo the ideal generated by $a-\lambda(a)$ for $a \in D_{\lambda} \subset \operatorname{ker}_{R} \partial \subset R[h]$. Then there exists a (unique) vertex algebra isomorphism map $f_{\lambda}$ from $\mathcal{V}_{\lambda}(R)$ to $\mathcal{V}_{h}^{\lambda}(R) /(h-1) \mathcal{V}_{h}^{\lambda}(R)$ such that

$$
f_{\lambda} \psi_{\lambda}(a)=a+(h-1) \mathcal{V}_{h}^{\lambda}(R) \quad \text { for } a \in R,
$$

where $\psi_{\lambda}$ is the quotient map from $\mathcal{V}(R)$ to $\mathcal{V}_{\lambda}(R)$. There exists a (unique) vertex Poisson algebra isomorphism map $g_{\lambda}$ from $S_{\lambda}(R)$ to $\mathcal{V}^{\lambda}(R) / h \mathcal{V}_{h}^{\lambda}(R)$ such that

$$
g_{\lambda} \phi_{\lambda}(a)=a+h \mathcal{V}_{h}^{\lambda}(R) \quad \text { for } a \in R,
$$

where $\phi_{\lambda}$ is the quotient map from $S(R)$ to $S_{\lambda}(R)$.

Remark 5.13 Let $R=\mathbb{C}[\partial] \otimes \mathfrak{g} \oplus \mathbb{C} c$ be the vertex Lie algebra associated with a Lie algebra $\mathfrak{g}$ equipped with a symmetric invariant bilinear form $\langle\cdot, \cdot\rangle$ (see Example 3.13 ). We have $\operatorname{ker}_{R} \partial=\mathbb{C} c$. Let $\ell$ be any complex number and let $\lambda_{\ell}$ be the linear functional on $\mathbb{C} c$ such that $\lambda_{\lambda}(c)=\ell$. Then $\mathcal{V}_{\lambda_{\ell}}(R)$ is isomorphic to $V_{\hat{\mathfrak{g}}}(\ell, 0)$ as in [LL] and to $V_{\ell}(\mathfrak{g})$ as in $[\mathrm{FB}]$. In this special case, a version of Theorem 5.12 was obtained in [FB] (Theorem 15.3.2).

Motivated by Proposition 5.8 we introduce the following notion:

Definition 5.14 Let $A$ be a vertex Poisson algebra and let $V$ be a vertex algebra over $\mathbb{C}$. We say that $V$ is a deformation of $A$ if there exists a vertex algebra $P[h]$ over $\mathbb{C}[h]$ such that $P[h] / h P[h]$ is commutative, $P[h] / h P[h] \simeq A$ and $P[h] /(h-1) P[h] \simeq V$.

It follows from Proposition 5.8 that for any filtered vertex algebra $(V, E), V$ is a deformation of the associated vertex Poisson algebra $\operatorname{gr}_{E} V$. In view of Theorems 5.11 and 5.12 , vertex algebras $\mathcal{V}(R)$ and $\mathcal{V}_{\lambda}(R)$ are deformations of vertex Poisson algebras $S(R)$ and $S_{\lambda}(R)$, respectively.

The notion of formal deformation of an algebra (see [G]) naturally suggests the following notion of formal deformation of a vertex algebra:

Definition 5.15 Let $(V, Y, 1)$ be a vertex algebra. A formal deformation of $V$ is a $\mathbb{C}$ bilinear map $Y_{h}$ from $V \otimes V$ to $V[[h]]\left[\left[x, x^{-1}\right]\right]$ such that $\left(V[[h]], Y_{h}, \mathbf{1}\right)$ carries the structure of an $h$-adic vertex algebra over $\mathbb{C}[[h]]$, where $Y_{h}$ extends canonically to a continuous $\mathbb{C}[[h]]$ bilinear map on $V[[h]] \otimes V[[h]]$, such that for $u, v \in V$,

$$
Y_{h}(u, x) v=Y(u, x) v+h Y_{1}(u, x) v+h^{2} Y_{2}(u, x) v+\cdots,
$$

where $Y_{i}$ are linear maps from $V$ to $\operatorname{Hom}(V, V((x)))$. 
One always has the $h$-adic vertex algebra structure over $V[[h]]$ with $Y_{h}$ being the natural extension of the vertex operator map $Y$ on $V$. This deformation is called the trivial deformation. Two formal deformations $Y_{h}^{(1)}$ and $Y_{h}^{(2)}$ on $V$ are said to be equivalent if there exists a continuous $\mathbb{C}[[h]]$-linear isomorphism $F$ of $V[[h]]$ such that $F(\mathbf{1})=\mathbf{1}$, $F(v) \in v+h V[[h]]$ for $v \in V$ and such that

$$
F\left(Y_{h}^{(1)}(u, x) v\right)=Y_{h}^{(2)}(F(u), x) F(v) \quad \text { for } u, v \in V .
$$

In particular, $F$ is an $h$-adic-vertex-algebra isomorphism from $\left(V[[h]], Y_{h}^{(1)}, \mathbf{1}\right)$ to $\left(V[[h]], Y_{h}^{(2)}, \mathbf{1}\right)$. The $\mathbb{C}[[h]]$-linear isomorphism $F$ necessarily has the form

$$
\Psi=1+h f_{1}+h^{2} f_{2}+\cdots
$$

with $f_{n} \in \operatorname{End}_{\mathbb{C}} V$ for $n \geq 0$. A vertex algebra $V$ is said to be rigid (in the category of vertex algebras) if any formal deformation is equivalent to the trivial deformation.

Remark 5.16 In the classical case, rigidity is closely related to the complete reducibility of certain modules. In [Z1-2] (cf. [FZ]), a notion of rationality of a vertex operator algebra $V$ was introduced in terms of the complete reducibility of certain $V$-modules (cf. [DLM1]). In principle, one should be able to show that rational vertex operator algebras in the sense of [Z1-2] are rigid in the category of vertex algebras.

Remark 5.17 In view of certain commutativity and associativity properties (see [FLM], [FHL], [DL], [Li2]), vertex algebras are analogous to commutative associative algebras (cf. [B2]). Certain noncommutative analogues of the notion of usual vertex algebra were studied in [B2], $[\mathrm{BK}]$ and [Li4], where usual vertex algebras are the commutative objects in a certain sense. Quantum vertex operator algebras studied in [EK] can be considered certain formal deformations of vertex operator algebras in the category of those noncommutative analogues of vertex algebras.

Let $Y_{h}$ be a formal deformation on a vertex algebra $V$. Since 1 is also the vacuum vector of the $h$-adic vertex algebra, from Proposition 5.3 we have $Y_{h}(\mathbf{1}, x)=1$, that is, $Y_{i}(\mathbf{1}, x)=0$ for $i \geq 1$. We also have $Y_{h}(v, x) \mathbf{1} \in V[[h, x]]$. In general, $Y_{h}(v, x) \mathbf{1}$ may depend on $h$. Now we assume that

$$
Y_{h}(v, x) \mathbf{1} \in V[[x]] \quad \text { for } v \in V,
$$

i.e.,

$$
Y_{i}(v, x) \mathbf{1}=0 \quad \text { for } v \in V, i \geq 1 .
$$

Then $Y_{h}(v, x) \mathbf{1}=Y(v, x) \mathbf{1}=e^{x \mathcal{D}} v$. Since $Y_{h}(v, x) \mathbf{1}=e^{x \mathcal{D}_{h}} v$ for $v \in V[[h]]$, the $\mathcal{D}$-operator $\mathcal{D}_{h}$ of $V[[h]]$ on $V$ agrees with the $\mathcal{D}$-operator $\mathcal{D}$ of $V$. Thus, $\mathcal{D}_{h}$ is the natural $\mathbb{C}[[h]]$-linear extension of $\mathcal{D}$. From the skew symmetry of the vertex algebra $V[[h]]$ we obtain

$$
Y_{n}(u, x) v=e^{x \mathcal{D}} Y_{n}(v,-x) u \quad \text { for } u, v \in V, n \geq 0 .
$$

Thus we have: 
Proposition 5.18 Let $Y_{h}$ be a formal deformation of a vertex algebra $V$ with the property

$$
Y_{h}(v, x) \mathbf{1} \in V[[x]] \quad \text { for } v \in V .
$$

Then

$$
\begin{aligned}
& Y_{h}(\mathbf{1}, x) v=v \\
& Y_{h}(v, x) \mathbf{1}=e^{x \mathcal{D}} v \\
& Y_{n}(u, x) v=e^{x \mathcal{D}} Y_{n}(v,-x) u \quad \text { for } u, v \in V, n \geq 0
\end{aligned}
$$

where $\mathcal{D}$ is the $\mathcal{D}$-operator of vertex algebra $V$.

Remark 5.19 We here recall the notion of $*$-deformation (see [BFFLS], [BW]) to compare with the vertex analogue. Let $A$ be a unital commutative associative algebra (over $\mathbb{C}$ ) and let $*_{h}$ be a family of associative multiplications on $A$ given by a formal power series

$$
a *_{h} b=\sum_{j \geq 0} B_{j}(a, b) h^{j}
$$

where each $B_{j}: A \times A \rightarrow A$ is a bilinear map. Then $*_{h}$ is called a $*$-deformation of $A$ if for $a, b, c \in A$,

1. $B_{0}(a, b)=a b$ (the product in $\left.A\right)$.

2. $B_{j}(b, a)=(-1)^{j} B_{j}(a, b)$

3. $B_{j}(1, a)=0$ for $j \geq 1$

4. $B_{i}$ are bidifferential operators (i.e., bilinear maps $A \times A \rightarrow A$ are differential operators with respect to each argument of globally bounded order).

5. $\left(a *_{h} b\right) *_{h} c=a *_{h}\left(b *_{h} c\right)$.

In view of Proposition 5.18 and Remark 5.19, we call a formal deformation $Y_{h}$ of a vertex algebra $V$ with the property (5.46) a $*$-deformation of $V$. Furthermore, motivated by Frenkel and Ben-Zvi's proposition (Proposition 5.6) we define the following notion:

Definition 5.20 Let $\left(A, Y_{-}, \partial\right)$ be a vertex Poisson algebra (over $\left.\mathbb{C}\right)$. A $*$-deformation of $A$ is an $h$-adic vertex algebra structure $\left(Y_{h}, \mathbf{1}\right)$ on $A[[h]]$ with $\mathbf{1}=1$ such that $Y_{h}(a, x) 1 \in$ $A[[x]]$ for $a \in A$ and such that for $a, b \in A$,

$$
Y_{h}(a, x) b=\left(e^{x \partial} a\right) b+h Y_{1}(a, x) b+O\left(h^{2}\right),
$$

where

$$
Y_{-}(a, x) b=\operatorname{Sing}_{x} Y_{1}(a, x) b \quad \text { for } a, b \in A .
$$


Remark 5.21 Let $Y_{h}$ be a *-deformation of a vertex Poisson algebra $\left(A, Y_{-}, \partial\right)$. Then

$$
\begin{aligned}
& \left(e^{x \partial} a\right) b=\lim _{h \rightarrow 0} Y_{h}(a, x) b \\
& Y_{-}(a, x) b=\operatorname{Sing}_{x} Y_{1}(a, x) b=\lim _{h \rightarrow 0} \frac{1}{h} \operatorname{Sing} Y_{h}(a, x) b \quad \text { for } a, b \in A .
\end{aligned}
$$

Furthermore, we have

$$
\begin{aligned}
\frac{1}{h}\left[Y_{h}\left(a, x_{1}\right), Y_{h}\left(b, x_{2}\right)\right] & =\operatorname{Res}_{x_{2}} x_{0}^{-1} \delta\left(\frac{x_{1}-x_{0}}{x_{2}}\right) \frac{1}{h} Y_{h}\left(Y_{h}\left(a, x_{0}\right) b, x_{2}\right) \\
& =\operatorname{Res}_{x_{2}} x_{0}^{-1} \delta\left(\frac{x_{1}-x_{0}}{x_{2}}\right) \frac{1}{h} Y_{h}\left(\operatorname{Sing} Y_{h}\left(a, x_{0}\right) b, x_{2}\right) .
\end{aligned}
$$

As with Poisson algebras, the fundamental problem is about the existence, uniqueness and construction of $*$-deformation of each vertex Poisson algebra.

\section{References}

[BK] B. Bakalov and V. G. Kac, Field algebras, math.QA/0204282.

[BW] S. Bates and A. Weinstein, Lectures on the Geometry of Quantization, Berkeley Mathematics Lecture Notes, Vol. 8, 1997.

[BFFLS] F. Bayen, M. Flato, C. Fronsdal, A, Lichnerowicz, D. Sternheimer, Deformation theory and quantization, I. Deformation of symplectic structures, Ann. Physics 111 (1978), no. 1, 61-110.

[BD] A. A. Beilinson and V. Drinfeld, Chiral algebras, unpublished manuscript.

[B1] R. E. Borcherds, Vertex algebras, Kac-Moody algebras, and the Monster, Proc. Natl. Acad. Sci. USA 83 (1986), 3068-3071.

[B2] R. E. Borcherds, Vertex algebras, in: Topological Field Theory, Primitive Forms and Related Topics (Kyoto, 1996), edited by M. Kashiwara, A. Matsuo, K. Saito and I. Satake, Progress in Math. 160, Birkhäuser, Boston, 1998, 35-77

[CG] N. Chriss and V. Ginzburg, Representation Theory and Complex Geometry, Birkhaüser, Boston-Basel-Berlin, 1997.

[Di] J. Dixmier, Enveloping Algebras, (The 1996 Printing of the 1977 English Translation), Graduate Studies in Mathematics, Vol. II, American Mathematical Society, 1996.

[DL] C. Dong and J. Lepowsky, Generalized Vertex Algebras and Relative Vertex Operators, Progress in Math. Vol. 112, Birkhaüser, Boston, 1993. 
[DLM1] C. Dong, H.-S. Li and G. Mason, Twisted representations of vertex operator algebras, Math. Ann. 310 (1998), 571-600.

[DLM2] C. Dong, H.-S. Li and G. Mason, Vertex Lie algebra, Poisson algebras and vertex operator algebras, in: Recent Developments in Infinite-Dimensional Lie Algebras and Conformal Field Theory, Proc. of an International Conference, May 23-27, 2000, University of Virginia, ed. by S. Berman, P. Fendley, Y.-Z. Huang, K. Misra and B. Parshall, Contemporary Math. 297, Amer. Math. Soc., 2002, 69-96.

[EF] B. Enriquez and E. Frenkel, Geometric interpretation of the Poisson structure in affine Toda field theories, Duke Math. J. 92 (1998), 459-495.

[EK] P. Etingof and D. Kazhdan, Quantization of Lie bialgebras, V, Selecta Math. (N.S.) 6 (2000), 105-130.

[FFR] Alex J. Feingold, Igor B. Frenkel and John F. X. Ries, Spinor Construction of Vertex Operator Algebras, Triality, and $E_{8}^{(1)}$, Contemporary Math. 121 (1991).

[FB] E. Frenkel and D. Ben-Zvi, Vertex Algebras and Algebraic Curves, Mathematical Surveys and Monographs, Vol. 88, Amer. Math. Soc., 2001.

[FHL] I. Frenkel, Y.-Z. Huang and J. Lepowsky, On axiomatic approaches to vertex operator algebras and modules, Memoirs Amer. Math. Soc. 104, 1993; preprint, 1989.

[FLM] I. Frenkel, J. Lepowsky and A. Meurman, Vertex Operator Algebras and the Monster, Pure and Appl. Math. Vol. 134, Academic Press, Boston, 1988.

[FZ] I. B. Frenkel and Y. Zhu, Vertex operator algebras associated to representations of affine and Virasoro algebras, Duke Math. J. 66 (1992), 123-168.

[G] M. Gerstenhaber, On the deformation of rings and algebras: II, Ann. of Math. 84 (1966), 1-19.

[K] V. G. Kac, Vertex Algebras for Beginners, University Lecture Series 10, Amer. Math. Soc., 1997.

[KL] M. Karel and H.-S. Li, Certain generating subspaces for vertex operator algebras, J. Alg. 217 (1999), 393-421.

[Ko] M. Kontsevich, Deformation quantization of Poisson manifolds, I, q$\operatorname{alg} / 9709040$.

[LL] J. Lepowsky and H.-S. Li, Introduction to vertex operator algebras and their representations, Monograph, to appear. 
[Li1] H.-S. Li, Representation theory and tensor product theory for vertex operator algebras, Ph.D. thesis, Rutgers University, 1994.

[Li2] H.-S. Li, Local systems of vertex operators, vertex superalgebras and modules, J. Pure Appl. Alg. 109 (1996), 143-195; hep-th/9406185.

[Li3] H.-S. Li, Some finiteness properties of regular vertex operator algebras, J. Alg. 212 (1999), 495-514.

[Li4] H.-S. Li, Axiomatic $G_{1}$-vertex algebras, Commun. Contemporary Math., to appear.

[Lia] B.-H. Lian, On the classification of simple vertex operator algebras, Commun. Math. Phys. 163 (1994), 307-357.

[MP] A. Meurman and M. Primc, Annihilating Fields of Standard Modules of $\tilde{s l}(2, \mathbb{C})$ and Combinatorial Identities, Memoirs Amer. Math. Soc. 652, 1999.

[P] M. Primc, Vertex algebras generated by Lie algebras, J. Pure Appl. Alg. 135 (1999), 253-293.

[Z1] Y.-C. Zhu, Vertex operator algebras, elliptic functions and modular forms, Ph.D. thesis, Yale University, 1990.

[Z2] Y.-C. Zhu, Modular invariance of characters of vertex operator algebras, J. Amer. Math. Soc. 9 (1996), 237-302. 